\title{
LA REFORMA AGRARIA CHILENA: HECHOS ESTILIZADOS A LA LUZ DE UNA NUEVA BASE DE DATOS
}

\author{
José I. Cuesta \\ Universidad de Chicago \\ Francisco A. Gallego \\ P. Universidad Católica de Chile

\section{Guillermo Marshall}

Universidad de Illinois

\author{
José G. Díaz \\ P. Universidad Católica de Chile \\ Felipe González \\ Universidad de California (Berkeley)
}

José I. Cuesta. Estudiante de PhD en economía en la Universidad de Chicago. Email: jicuesta@uchicago.edu.

José G. DíAz. Profesor del Instituto de Economía e investigador del EH Clio Lab de la UC. Email: jdiazb@uc.cl.

Francisco A. Gallego. Profesor del Instituto de Economía e investigador del EH Clio Lab de la UC. Email: fgallego@uc.cl.

Felipe GonzÁlez. PhD en economía por la University de California en Berkeley. Email: fel@berkeley.edu.

Guillermo Marshall. Profesor de economía en la Universidad de Illinois en Urbana-Champaign. Email: gmarshll@illinois.edu.

* Los autores quieren señalar: "Agradecemos los comentarios y sugerencias recibidos de Alberto Valdés (coeditor) y de los dos árbitros anónimos de Estudios Públicos, así como agradecemos a Amanda Dawes y a Daniela Marshall por su excelente asistencia en la investigación, al personal de los archivos del Cora y a Bartolomé Payeras, del INE, por el acceso a los datos. La investigación ha sido financiada por Conicyt/Programa de Investigación Asociativa SOC 1102. Mantenemos el monopolio de los errores". 
RESUMEN: Este artículo presenta un conjunto de hechos estilizados para la reforma agraria chilena a partir de datos provenientes de registros de cada predio involucrado. La información considera tamaño y ubicación de los predios, el momento de las expropiaciones, las razones legales invocadas para la expropiación y el resultado final del proceso. El artículo también presenta medidas de la intensidad de la reforma a nivel comunal y su correlación con la producción agrícola y la estructura de propiedad de la tierra antes de la reforma. Los antecedentes presentados confirman algunos rasgos conocidos del proceso de reforma y sugieren nuevas dimensiones: se identifica una alta heterogeneidad en el tamaño de los predios expropiados, así como en la intensidad de la reforma y de la contrarreforma a nivel comunal. Además, el examen de los datos sugiere que los problemas de desigualdad y de tenencia de la tierra fueron, en la práctica, motivaciones importantes de la reforma agraria.

Palabras Clave: reforma agraria, Eduardo Frei Montalva, Salvador Allende, microdatos, hechos estilizados.

RECIBIDO: enero 2017; ACEPTADO: junio 2017.

JEL: C80, N56, Q15,

\section{CHILE'S AGRARIAN REFORM: STYLIZED FACTS IN THE LIGHT OF A NEW DATABASE}

ABstract: This paper presents a series of stylized facts for the Chilean agrarian reform building on data from archival files of each plot involved in the process. The data includes the size and location of each plot, the time of the expropriations, the legal reasons invoked for the expropriation and the final result of the process. The paper also presents measures of the intensity of the reform at the municipality level and the correlation with agricultural production and the structure of ownership before the reform. While the results confirm some previous stylized facts of the reform process, we also provide new evidence that helps to understand the main characteristics of the process: there is a high heterogeneity in the size of expropriated plots as well as in the intensity of reform and the counter-reform process at the municipality level. Moreover, the data also suggest that the aspects related to land ownership were important motivations of the agrarian reform.

Keywords: agrarian reform, Eduardo Frei Montalva, Salvador Allende, microdata, stylized facts.

RECEIVED: January 2017; ACCEPTED: June 2017.

JEL: C80, N56, Q15. 


\section{INTRODUCCIÓN}

a reforma agraria chilena sigue siendo hoy objeto de controversia. Esto queda en evidencia al examinar el debate en la prensa de Chile a propósito de los 50 años de la reforma de Frei. ${ }^{1}$ La controversia incluye tanto al diagnóstico inicial que motivó la reforma como a los medios empleados para su desarrollo, sus efectos y sus alcances.

La visión positiva de la reforma destaca que la motivación fue terminar con la baja productividad del latifundio y con el sistema de inquilinaje que era considerado la causa del atraso en el campo chileno. Sin tales transformaciones, se postula, no se explicaría el desempeño actual del sector agrícola (Moreno 2013; 2016; Cox 2016). En un sentido similar, Bengoa $(2015,338)$ destaca que la reforma generó "la creación de un mercado de tierras como consecuencia de la pulverización de la propiedad".

En cambio, la visión crítica de la reforma agraria cuestiona el diagnóstico inicial, por subestimar el papel de las regulaciones en el sector que desincentivaban la inversión. Igualmente discute la eficiencia de los medios utilizados, especialmente los procedimientos de expropiación y los mecanismos de reasignación de las tierras para su explotación, que no necesariamente se tradujeron en propiedad campesina. Finalmente, se pone en entredicho los alcances de la reforma, puesto que los ajustes del gobierno militar revirtieron parcialmente las medidas (Arnello 2016; Crespo 2016; Valdés 2016a; 2016b; Williamson 2016). Valdés (2016a; 2016b) señala que la preocupación por el desempeño productivo era importante, pero no constituía la motivación central de la reforma, y las principales transformaciones del sector ocurrieron después de (y no durante) el proceso. Lo principal habría sido favorecer a los campesinos asalariados para reducir su dependencia del patrón y acabar con la captura de su voto (Fermandois 2016).

En este punto, cabe mencionar las razones por las cuales la experiencia chilena es interesante. En primer lugar, es un ejemplo de las reformas agrarias implementadas en América Latina a partir de la década de 1960 en el contexto de la llamada Alianza para el Progreso, un

${ }^{1}$ El debate comienza con la columna de Williamson (2016) que comenta el trabajo de Valdés y Foster (2014), y sigue con una serie de respuestas, entre otras de Arnello (2016), Cox (2016), Crespo (2016), Moreno (2016) y Valdés (2016a). 
ambicioso programa de ayuda patrocinado por Estados Unidos, cuyo fin principal era mejorar las condiciones sociales de la región (Edwards 2009; González 2013). En segundo lugar, el caso chileno es llamativo debido a la existencia de una estructura tradicional de la propiedad agraria muy concentrada (véase para más detalles Bauer 1975; Bellisario 2007a; 2007b; Baland y Robinson 2008). En tercer lugar, la reforma fue un proceso iniciado y ejecutado principalmente por gobiernos democráticos, en contraste con los ejemplos bien conocidos de las reformas agrarias implementadas en algunos países del sudeste asiático (Valdés y Foster 2014; 2015). Además, como Bellisario (2007a; 2007b) plantea, tres gobiernos con orientaciones políticas muy diferentes utilizaron fundamentalmente el mismo marco legal, aunque con diferentes objetivos políticos y con resultados muy dispares en términos de la intensidad y profundidad de la reforma. ${ }^{2}$ Esto implica que la implementación de la reforma se relacionaría fundamentalmente con decisiones discrecionales de los diferentes gobiernos, un fenómeno que vale la pena estudiar empíricamente. Por último, el caso de Chile también es interesante porque el país experimentó con varios cambios económicos y sociales relacionados con otras dimensiones y, por lo tanto, puede ser interesante estudiar cómo la reforma agraria interactúa con estos otros cambios de políticas.

Considerado lo anterior, y como señala Banerjee (1999), no es de extrañar que la discusión sobre los efectos en eficiencia y en equidad de la reforma agraria presente tanto una resonancia emocional como una naturaleza multifacética. En este contexto, el uso de antecedentes y datos detallados puede facilitar la comprensión científica de las causas y dilucidar las consecuencias del proceso en cuestión. ${ }^{3}$

${ }^{2}$ Aunque la primera ley de reforma data de 1962, la ley promulgada en 1965 fue el instrumento principal del proceso. Los tres gobiernos incluyen el gobierno reformista de centro-izquierda liderado por el demócrata cristiano Eduardo Frei Montalva (1964-1970), el gobierno comunista-socialista liderado por Salvador Allende (1970-1973) y la dictadura militar de Augusto Pinochet (1973-1990). La primera ley de reforma fue dictada durante el gobierno de derecha de Jorge Alessandri (1958-1964).

${ }^{3}$ Aquí se puede mencionar la idea de la llamada "relación inversa", que plantea que, en los países en desarrollo, la productividad de la tierra decrecería a medida que aumenta el tamaño de los predios. Esta idea fue planteada originalmente por Sen (1962) y ha sido largamente discutida y cuestionada en base a posibles errores de medición (Barbier 1984; Gaurav y Mishra 2015). Ver Banerjee (1999) para una discusión más detallada al respecto. 
Para contribuir a los objetivos mencionados, en este trabajo se explora una serie de hechos estilizados del fenómeno a partir del empleo de una nueva base de datos que proporciona información detallada a nivel nacional y de predios agrícolas. ${ }^{4}$ Estos antecedentes permitirán examinar mejor el proceso de la reforma agraria chilena, facilitando a investigadores de diferentes ciencias sociales la búsqueda de respuestas a preguntas relacionadas con sus causas, sus consecuencias (sobre diferentes aspectos) y su interacción con otros cambios en las políticas públicas. $^{5}$

El proceso para construir la base de datos se inició con la digitalización de aproximadamente 5.700 archivos que registraban todos los predios agrícolas que participaron del proceso de reforma agraria. Cada archivo resume la evolución de los movimientos de la propiedad (expropiaciones y devoluciones a los propietarios originales) para cada predio involucrado en el proceso. Los archivos presentan información formal del predio agrícola, incluyendo su nombre, propietario y su ubicación geográfica. A continuación, utilizando la información básica construimos medidas de la intensidad del proceso de reforma agraria entre 1965 y 1975 . Esta variable mide el porcentaje de tierra que ingresó al proceso de reforma, tanto en el país como a nivel de comunas. ${ }^{6}$

Con estos elementos identificamos una serie de hechos estilizados del proceso de reforma agraria, tanto a nivel país como a nivel comunal. Destacamos aquí un grupo de resultados novedosos (compárese con Garrido 1988: Bellisario 2007a: 2007b; Valdés y Foster 2014). En primer lugar, encontramos que el tamaño medio de los predios agrícolas que entraron en el proceso de expropiación fue de alrededor de 1.800 hectáreas físicas (HF). Si bien este número es similar a los promedios reportados en Bellisario (2007a) y Garrido (1988), la disponibilidad de

${ }^{4}$ Los datos están disponibles en la página web de Economic History and Cliometrics Lab (EH Clio Lab). URL: http://cliolab.economia.uc.cl/.

${ }^{5}$ Valdés y Foster $(2014 ; 2015)$ argumentan que la reforma agraria chilena incluye no sólo la reasignación de tierras, sino también otras políticas relacionadas con cambios tanto en la estructura agraria como en los incentivos para los agentes que operan en el sector. Aunque seguimos la literatura y utilizamos el término "reforma agraria", nuestros datos sólo incluyen información sobre el primero de los componentes mencionados (o sea, la reasignación de tierras).

${ }^{6}$ Como se describe en el anexo, en este trabajo utilizamos 271 comunas comparables intertemporalmente desde la década de los 1960. Los supuestos utilizados se indican de manera explícita. 
microdatos (o sea, información a nivel de cada predio) permite estudiar las características de los predios con mucho más detalle. Por ejemplo, la mediana del tamaño de los predios agrícolas que entraron en el proceso fue sólo de $385 \mathrm{HF}$. Asimismo, los nuevos datos permiten identificar que el percentil 25 de la distribución de tamaño era de cerca de $150 \mathrm{HF}$. Esto implica que el mayor valor del promedio resulta de la existencia de propiedades muy grandes que entraron en el proceso. Además, los microdatos también permiten concluir que para 1973 el 91,4 por ciento de los predios agrícolas que entraron en el proceso estaban completamente expropiados.

En segundo lugar, en promedio, alrededor del 20 por ciento de la tierra de las comunas entró al proceso de expropiación. Una vez más nuestra base de datos permite identificar un relativamente alto grado de heterogeneidad entre comunas, pues, mientras la distribución se concentra entre 0 y 20 por ciento, hay comunas en las que la cifra llega a un número tan alto como 95,4 por ciento. Esto implica que existe de facto una heterogeneidad en la intensidad con la que se llevó a cabo el proceso de expropiación en las diferentes zonas del país. A su vez, un nuevo resultado que desafía la mirada convencional acerca de la intensidad de la reforma es que esta heterogeneidad que se observa a nivel comunal no parece estar correlacionada con variables geográficas o con proxies de productividad de la tierra, pero sí con medidas que capturan la estructura de la propiedad de la misma, como el nivel inicial de desigualdad en la propiedad de la tierra. Como consecuencia de ello, nuestros datos sugieren que el llamado proceso de contrarreforma (que comenzó en 1973) también presenta un alto grado de heterogeneidad entre diferentes regiones del país. Este conjunto de resultados a nivel comunal es nuevo en la literatura y muestra el uso potencial de la base de datos presentada en este artículo.

En tercer lugar, en cuanto al momento de la reforma, nuestros datos confirman resultados anteriores que señalan al período 1970-1973 como la etapa más intensiva del proceso de reforma agraria. ${ }^{7}$ En este período se observa un cambio tanto en el número de expropiaciones

${ }^{7}$ Un factor que contribuye a explicar la aceleración del proceso de reforma agraria incluso al finalizar el gobierno de Frei fue la Ley 17.280 (llamada Ley Aylwin), que permitía a la CORA tomar posesión inmediata del fundo en expropiación y reducía la posibilidad de recurrir a tribunales (Fontaine 2001). 
como en el tipo de predio expropiado, ya que predios mucho más pequeños entraron al proceso. Esto induce una disminución tanto en la media como en la mediana del tamaño de los predios agrícolas expropiados.

Respecto a los motivos legales de expropiación, documentamos que también existe una variación significativa en esta dimensión. La razón que invocaba que el predio era "demasiado grande" aumentó su relevancia a través de los períodos y terminó siendo la razón más importante para expropiar. A su vez, mientras la razón "explotación ineficiente del predio" alcanzó relevancia al final del proceso, la razón "predio ofrecido por el propietario a la agencia gubernamental" tuvo más peso en el inicio, lo que sugiere que los propietarios asumieron que la expropiación era inevitable y era mejor recibir una compensación luego de ofrecer voluntariamente el predio.

Respecto al proceso que Bellisario (2007a; 2007b) denomina contrarreforma parcial y que se produce durante la dictadura militar, se implementó un tipo diferente de reforma que, básicamente, detuvo el proceso de expropiación sin revertir lo ejecutado y trató de regularizar la situación de los predios agrícolas expropiados (la mayoría de los cuales no tenían dueños y eran manejados por cooperativas o directamente por el gobierno). En este sentido, los datos de nuestra base sugieren que la distribución de la propiedad a los campesinos que habían sido beneficiados inicialmente por la reforma y la revocación de las expropiaciones ilegales fueron los principales mecanismos para asignar derechos de propiedad.

La información utilizada en este trabajo permite relacionar, para cada predio, el motivo legal para las expropiaciones con el estado final del mismo. Encontramos que, para la mayoría de las causas legales, la mayor parte de las expropiaciones fueron asignadas y que una proporción no trivial fue revocada, regresando la propiedad a sus dueños originales. Este resultado general no se observa para el caso de los predios agrícolas que fueron expropiados invocando que estaban siendo trabajados ineficientemente o abandonados. Para esta causal, el número y superficie de los predios agrícolas revocados es mucho mayor que el número de los distribuidos. Esto apoya la hipótesis de Bellisario (2007a), quien plantea que la naturaleza discrecional de esta causa legal permitió su uso para justificar expropiaciones. 
Estos hechos estilizados mencionados ilustran dos contribuciones de la nueva información disponible en la base de datos utilizada en este trabajo. Primero, mientras investigaciones anteriores, como Garrido (1988) y Bellisario (2007a; 2007b), presentan un resumen estadístico del proceso de reforma agraria usando las mismas fuentes primarias (los archivos de la Corporación de Reforma Agraria, CORA), nuestros microdatos aportan antecedentes a nivel de predio agrícola en un formato digitalizado, lo que permite identificar patrones que van más allá de promedios y medir heterogeneidad examinando correlaciones más detalladas.

Un segundo aporte de esta base de datos a la literatura es la construcción de proxies para la intensidad de las expropiaciones a nivel de comunas. Esto permite un examen más preciso de la implementación de facto de la reforma agraria, más aún si se considera que los hechos estilizados presentados sugieren que la reforma agraria en Chile fue un proceso con una heterogeneidad significativa entre comunas y en el tiempo. ${ }^{8}$ Futuras investigaciones podrían estudiar con más detalle la variación de facto de la intensidad de la reforma en diferentes comunas utilizando el nuevo conjunto de datos presentados en este artículo.

El resto del artículo se organiza de la siguiente manera. La sección 2 describe brevemente el proceso de reforma agraria chilena. La sección 3 presenta la metodología utilizada para construir la base de datos de este trabajo. La sección 4 muestra una serie de hechos estilizados derivados de este conjunto de datos y la sección 5 concluye brevemente.

\section{LA REFORMA AGRARIA CHILENA}

Con el fin de entender las causas y posibles consecuencias del proceso de reforma agraria, es importante entender el papel de la agricultura en la historia de Chile. Para ello, aquí partimos presentando una revisión simplificada de la evolución de la agricultura en la historia de

${ }^{8}$ Bellisario (2007a) sostiene que una de las características más interesantes de la reforma agraria chilena es que, usando la misma ley, gobiernos con muy diferentes ideologías implementaron distintos tipos de reforma agraria (tanto en términos de intensidad como de otras características). Sin embargo, en estricto rigor no hubo una ley, sino una evolución de instrumentos legales. 
Chile. ${ }^{9}$ En el momento de la Independencia, el campo chileno tradicional comprendía la zona situada entre el valle de Aconcagua y el río Maule. La propiedad agrícola estaba muy concentrada y tuvo su origen en concesiones de tierras hechas en el período colonial ("mercedes de tierra"). ${ }^{10}$ Esta estructura agraria, a veces denominada sistema de hacienda, no fue alterada por los cambios políticos siguientes, aunque en la década de 1850 los mayorazgos fueron abolidos (Bauer 1975).

Desde mediados del siglo XIX, Chile enfrentó una creciente demanda internacional por productos de la agricultura. Los principales efectos de este aumento de la demanda fueron un incremento de las exportaciones de granos y la expansión de la tierra cultivada hacia el sur en el territorio de La Araucanía (la "Frontera"). Los productos agrícolas se exportaron a algunos mercados donde hubo una demanda temporal - como California y Australia durante su "fiebre del oro"- y también a mercados con demanda más permanente para los granos, como Inglaterra y las regiones mineras chilenas. Collier y Sater $(2004,265)$ afirman que Chile era "la primera nación agrícola en la costa del Pacífico de América del Sur".

Es materia de controversia qué sucedió con la agricultura chilena a principios del siglo XX. Algunos autores hacen hincapié en el papel de los factores externos para explicar la desaceleración de la agricultura chilena: la aparición de nuevos y grandes competidores (Australia, Canadá, Estados Unidos, Argentina y Rusia) que bajaron los precios internacionales, y las crisis ocasionadas por la apertura del Canal de Panamá y la Gran Depresión. Otros señalan los problemas locales como elementos que no permitieron mantener la competitividad: las cosechas no crecieron a la tasa observada en décadas anteriores debido a problemas climáticos, al agotamiento de la tierra o a la falta de modernización tecnológica en la agricultura.

${ }^{9}$ Meller (1996) y Hachette (2011) presentan discusiones sobre la historia de la política económica de Chile que son útiles de tener en cuenta al analizar la historia de la agricultura de Chile. A su vez, Barahona et al. (1988), Bauer (1975), Bengoa (2015), Cuesta et al. (2015), Loveman (1976), PUC (1976), Santana (2006) y Valdés (1994) presentan revisiones históricas del sector agrícola chileno considerando diferentes etapas e hipótesis.

${ }^{10}$ Chile sigue un patrón similar al observado en otras colonias españolas de América del Sur en la época de la Independencia. Véase Assadourian (2006) para más detalles. 
La agricultura chilena se consideró un "sector estancado" desde la década de 1930, una afirmación basada principalmente en dos indicadores: el bajo crecimiento de la producción agrícola (medido de diferentes maneras) y el saldo negativo del comercio de estos productos (Ballesteros 1965; Gómez y Echeñique 1998). Es cierto que la participación de la agricultura en el PIB de Chile se redujo del 14 por ciento en 1860 al 6 por ciento en 1907 y al 5 por ciento en 1952 (Díaz et al. 2016). Sin embargo, los trabajadores agrícolas eran todavía una parte importante de la fuerza de trabajo total en la década de 1950: mientras que en 1860 el 42 por ciento de la población activa total trabajaba en la agricultura, la cifra se redujo al 38 por ciento en 1907, y al 30 por ciento en 1952. Además, en 1930 todavía más de la mitad de la población chilena era rural (Díaz et al. 2016). En el contexto de una economía que pretendía reforzar su mercado doméstico, un sector agrícola débil fue visto como un obstáculo para la mejora de las condiciones de vida de la población.

Antes de la década de 1930 las políticas agrícolas chilenas proporcionaron mejoras generales a los productores. El gobierno de Chile era una fuente de crédito para los propietarios de tierras (a través de la Caja de Crédito Hipotecario), invirtió en programas de regadío y reguló la propiedad de nuevos asentamientos (a través de la Caja de Colonización Agrícola) (Brock 2009; Robles-Ortiz 2009). El Ministerio de Agricultura fue creado en 1924 (Ministerio de Agricultura, Industria y Colonización, el nombre actual data de 1930). Tras la Gran Depresión, sin embrago, las políticas agrícolas fueron cuestionadas debido a la crisis diagnosticada en el sector.

Las explicaciones para el estancamiento se pueden agrupar en dos categorías principales: por un lado, el sistema de tenencia de la tierra; por otro, las políticas públicas que afectaban a la agricultura. La primera explicación es la conjetura de que el estancamiento de la agricultura proviene de la estructura de tenencia de la tierra. En términos estilizados, ésta es una tesis tradicional que sostiene que las grandes haciendas o latifundios no invierten lo suficiente, reduciendo el crecimiento de la productividad y causando una oferta de productos agrícolas distorsionada. ${ }^{11}$ Esta última característica, combinada con un exceso de demanda, sería una fuente clave de la inflación chilena (Wachter 1979). Esta idea

${ }^{11}$ Esto se motiva por la idea de la "relación inversa" discutida en la introducción. 
fue promovida por la escuela estructuralista en los años 1950 y 1960 , aunque su planteamiento original se remonta a la década de 1930. Por ejemplo, el geógrafo George McBride (1936), hablando en Chile, sostenía que "en distintos aspectos la hacienda se administra hoy lo mismo que en la colonia. El latifundio es responsable del atraso de los métodos agrícolas". Como el 43 por ciento de la población activa agraria no poseía tierras en 1955 (Kay y Silva 1992), se propuso una serie de cambios: una reforma agraria que crease pequeñas unidades y la promoción de los derechos laborales entre los trabajadores agrícolas. Cabe señalar que este tipo de políticas también se vieron favorecidas por la política exterior estadounidense a través de la Alianza para el Progreso. ${ }^{12}$

La segunda explicación para el estancamiento de la agricultura se relaciona con los efectos de las políticas del gobierno que afectaban al sector directa e indirectamente (Almonacid 2009; Cuesta et al. 2015; Hachette 2011, Hurtado et al. 1990; Portilla 2000; Santana 2006). Desde la década de 1930 un conjunto de disposiciones restrictivas, controles de precios y regulaciones de oferta afectaron a la agricultura. Loveman $(1976 ; 2001)$ sugiere que estas medidas eran un curioso "acuerdo" político entre los propietarios de tierras, los trabajadores urbanos y los movimientos políticos reformistas. Mientras los trabajadores urbanos obtenían precios baratos de los alimentos, los propietarios retenían control político de los trabajadores rurales mediante la limitación de la aplicación de las leyes laborales. ${ }^{13}$ En la misma línea, Millar y Fernández (2008) sostienen que la creciente intervención del gobierno respondió a demandas inmediatas de grupos de presión en lugar de a objetivos a largo plazo, lo que afectó negativamente a los rendimientos en el sector agrícola.

De modo complementario, la literatura muestra cómo la existencia de una política comercial orientada a la sustitución de importaciones

${ }^{12}$ La siguiente cita refleja en buena medida esta postura: "La reforma agraria se trata a menudo como la panacea para los males de América Latina (...). La importancia de una reforma agraria reside más en su redistribución del poder que en su redistribución de la tierra” (Becket, 1963, 193). Ver también Barraclough (1970).

${ }^{13}$ Una fuerza de trabajo agrícola no sindicalizada podría afectar el desempeño de la agricultura chilena y el control de los propietarios sobre los votos de sus trabajadores (Lapp 2004, cap. 3). Baland y Robinson (2008) encuentran apoyo parcial a esta hipótesis. Lederman (2005) provee una explicación de economía política más general para las políticas comerciales que afectaban directa e indirectamente al sector agrícola en este período. 
produjo un patrón de desprotección efectiva para la agricultura (un patrón que por lo demás se repite en otros países, tal como documentan Krueger et al. 1988; 1991). Por ejemplo, Hachette (2011) señala que la agricultura enfrentaba una tasa de protección efectiva negativa de -27 por ciento para el período 1960-1969 (mientras que la contracara de esto era que el resto de los sectores enfrentaban una tasa de protección efectiva positiva de 73 por ciento). Valdés y Jara (2008) y Cuesta et al. (2015) presentan evidencia de los efectos de estas distorsiones en la asignación de recursos y resultados del sector agrícola. Valdés et al. (1990) confirman el impacto sobre la agricultura chilena del cambio en la estrategia de desarrollo tras la crisis económica de 1930, a través de los efectos vía tipo de cambio real.

En este contexto, la reforma agraria y la sindicalización de los trabajadores agrícolas surgieron entre las cuestiones más importantes de la década de 1960. El proceso chileno de reforma agraria comenzó de manera muy limitada en 1962 y progresó de forma constante desde 1964 hasta 1973, cuando las expropiaciones cesaron después de que la dictadura militar tomase el poder. Comenzó entonces la llamada contrarreforma, que continuó hasta 1980.

En términos más específicos, la reforma agraria chilena se puede dividir así en tres períodos definidos en términos generales (Huerta 1989; Bellisario 2007a; Valdés y Foster 2014; 2015). En el primer período (antes de 1965), el proceso fue un modesto intento por parte de una administración de derecha para fraccionar algunas propiedades de gran tamaño. En noviembre de 1962 el Congreso promulgó la Ley 15.020, que autorizó al Estado a comprar un terreno pagando hasta el 20 por ciento del valor en efectivo y el saldo con bonos que devengaban intereses con vencimiento en diez años. Esta ley creó una serie de agencias de gobierno, entre ellas la CORA y el Indap (Instituto de Desarrollo Agropecuario), para tareas de coordinación. Todo este proceso fue liderado por la CORA hasta que fue sustituida en 1978 por la Odena (Oficina Nacional de Normalización Agrícola). Finalmente, el SAG (Servicio Agrícola y Ganadero), creado en 1967, asumió las últimas labores relacionadas en 1989 .

El segundo período se inicia en 1965 y duró hasta septiembre de 1973. Según Kay (1978), mientras que el gobierno democratacristiano -Eduardo Frei, 1964-1970 - trató de modernizar y consolidar 
un modo de producción capitalista, el gobierno de la Unidad Popular -Salvador Allende, 1970-1973 - intentó destruir las relaciones capitalistas como un primer paso hacia la transición al socialismo. Después de una reforma constitucional que redefinió los derechos de propiedad (1965), en julio de 1967 fue aprobada la Ley 16.640, que amplió las causales de expropiación. Entre estas causales, las razones más importantes por las que un predio podría ser expropiado fueron (i) si su superficie superaba las 80 hectáreas de riego básico (HRB) ${ }^{14}$, (ii) si fue abandonado o se explotó de manera ineficiente, o (iii) si fue ofrecido por su propietario a la CORA, de acuerdo a los artículos 3, 4 y 10 de la Ley 16.640 (Garrido 1988). La compensación por una expropiación era con un pago parcial en efectivo y bonos del Estado a largo plazo. La tabla 1 presenta una lista completa de las causas legales.

Desde 1971, el gobierno de la Unidad Popular puso en marcha un programa de transición hacia el socialismo en Chile y la reforma agraria fue integrada a este proceso. Las expropiaciones de tierras se aceleraron, incluyendo ahora incluso predios por debajo de las $80 \mathrm{HRB}$ y el gobierno de Allende convirtió ocupaciones de tierras o huelgas ilegales en transferencias de facto de la responsabilidad administrativa de propiedades rurales (Loveman 2001). Mientras que la reforma del gobierno de Frei adoptó un modelo que promovía la instalación de cooperativas de productores ("asentamientos"), el ala radical de la UP buscó la creación de nuevas unidades agrícolas basadas en la propiedad estatal de la tierra. $^{15}$

Después de 1973, en el tercer período, el gobierno militar detuvo el proceso de reforma agraria. Sin embargo, Bellisario (2013) sostiene que, puesto que la tierra expropiada no fue totalmente devuelta a sus propietarios originales, hubo un período de contrarreforma "parcial" a partir de 1973 y la distribución de la tierra expropiada a muchos nuevos propietarios era parte de una nueva etapa en la transformación capitalista de la agricultura chilena.

${ }^{14}$ La HRB es una unidad de cuenta que pretendía estandarizar los tamaños de los predios agrícolas en función de su calidad, y permitía así a la CORA considerar no sólo el tamaño del predio, sino también su productividad al momento de determinar las expropiaciones.

${ }^{15}$ Collier y Sater $(2004,337)$ afirman: "La UP no tenía una concepción única sobre la forma en que debía ser reorganizado el campo en el futuro". 


\section{Tabla 1. CAUSAS LEGALES DE EXPROPIACIÓN}

Estas causales son las principales mencionadas en las fuentes de la CORA y la numeración de referencia procede de su asociación con artículos de la Ley 16.640. Ver Garrido (1988, 159-162 y su Anexo IV).

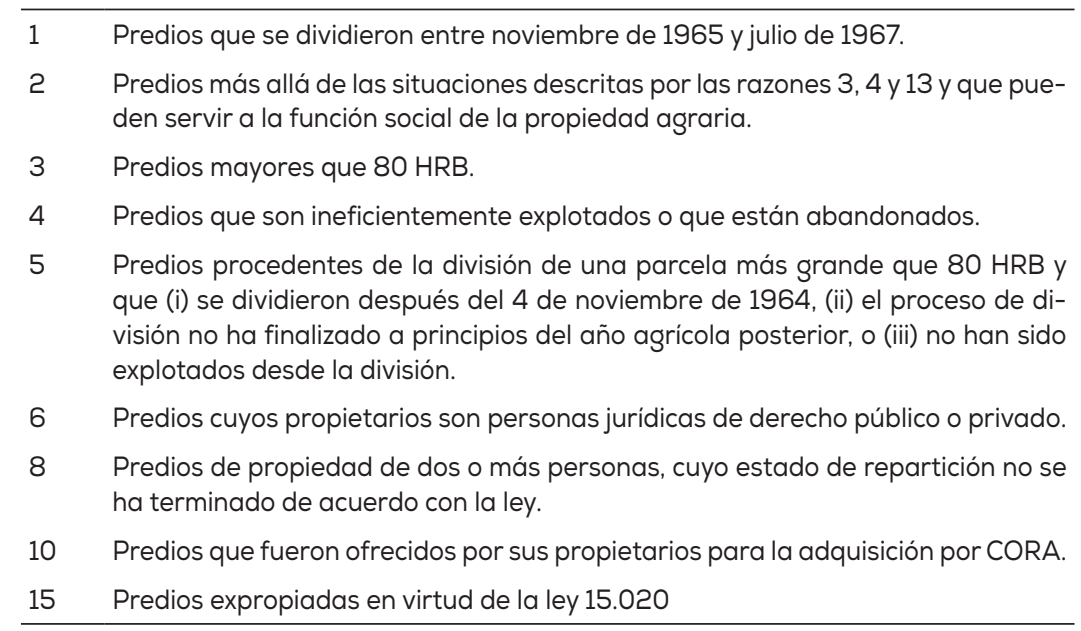

\section{METODOLOGÍA: CONSTRUCCIÓN DE BASE DE DATOS Y PRINCIPALES SUPUESTOS}

En esta sección se explica la metodología (definiciones, fuentes y supuestos) empleada para construir nuestra base de datos sobre la reforma agraria chilena. En la construcción de la base de datos sólo se usaron supuestos para la variable de intensidad; las otras variables utilizadas en el estudio provienen directamente de archivos. ${ }^{16}$

La Odena y el SAG conservan un grupo de archivos que incluyen todos los eventos relacionados con la reforma agraria a nivel de predios agrícolas. Estos archivos de la reforma agraria, que totalizan alrededor de 5.702 registros, son la principal fuente de datos que utilizamos. Cada archivo resume la evolución de las expropiaciones y las devoluciones para cada predio que entró en el proceso. Los archivos presentan antecedentes formales del predio, incluyendo su nombre, propietario y ubicación geográfica, así como todos los hitos en que el predio estuvo involucrado. La figura 1 presenta un ejemplo de uno de estos archivos.

${ }^{16}$ Los procedimientos empleados están en archivos do de STATA, disponibles junto con la base de datos. 
Figura 1. UN EJEMPLO DE LOS ARCHIVOS

a)

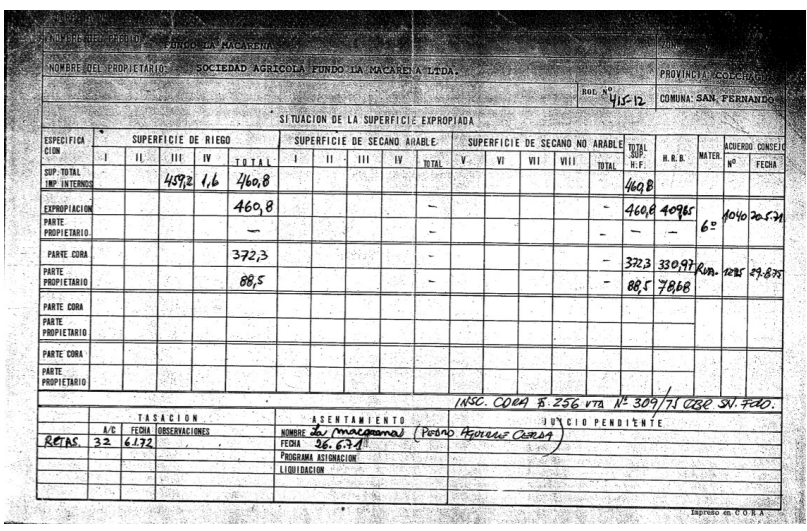

b)

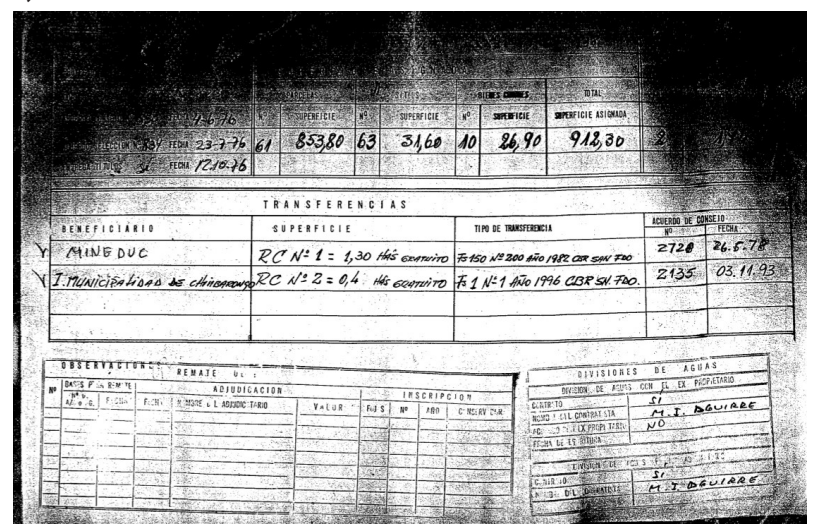

Específicamente, la descripción de los predios ofrecida por los archivos incluye las siguientes entradas:

a) una medición del predio llevada a cabo al comienzo del proceso de reforma por el Servicio de Impuestos Internos (SII), que reporta el tamaño y calidad de la tierra;

b) una descripción de la expropiación del predio agrícola, incluyendo el momento de la expropiación, la parte del predio que se dejó al propietario original, la parte que fue expropiada, la fecha en la que se ordenó la expropiación y la causa legal de la expropiación; y 
c) cada movimiento desde la expropiación inicial, ya sea por juicios posteriores con los propietarios originales o por nuevas expropiaciones ordenadas por la CORA (la información es presentada en el formato ya descrito).

Los registros incluyen dos unidades de medición para el tamaño del predio agrícola: las hectáreas físicas (HF) y las hectáreas de riego básico (HRB). Como se discutió más arriba, las HRB corresponden a una unidad de medida para estandarizar los tamaños de los predios considerando, también, su productividad. ${ }^{17}$

Utilizamos la información sobre tamaño del predio agrícola de la siguiente manera: se toma la medición del SII en el inicio del proceso y luego se consideran dos etapas adicionales: (i) el estado del predio después de la etapa de expropiación y (ii) el estado del predio después del proceso de contrarreforma, que incluye todos los movimientos observados hasta el estado final.

La principal limitación de los archivos de la CORA es que la información obtenida está completa sólo para algunos predios. Nosotros suplimos la información faltante en aquellos casos en los que había suficientes datos en el archivo para que una interpolación simple fuese razonable. En tan sólo 15 de los casos esto no fue posible y hemos dejado esta información como perdida. Además, los datos sobre calidad de los predios agrícolas no están disponibles más allá del reporte inicial del SII.

Otra dificultad es que en algunos casos no es posible tener información sobre el tamaño del predio medido en HRB y, dada la ausencia de descripciones geográficas de los predios agrícolas, no es posible llenar la información usando procedimientos alternativos.

Por último, el tamaño de los predios agrícolas reportados en los archivos varía a través del tiempo y crea ciertos desajustes. En este caso, se supuso que la calidad de los datos mejora con el tiempo y, por lo tanto, utilizamos las medidas más recientes disponibles en aquellos casos problemáticos.

En resumen, la base de datos incluye 5.687 predios agrícolas expropiados, que dan cuenta de 9.929.766 HF expropiadas a lo largo

${ }^{17}$ Los archivos también recogen información sobre (i) algunos beneficios dados por el gobierno a los expropiados y (ii) todas las transferencias de propiedad a otros agentes, incluidas las cantidades involucradas y las fechas. 
de todo el período en el que la reforma agraria estaba activa, y para 9.665.508 HF expropiadas entre el inicio del gobierno de Frei y el fin del gobierno de Allende.

A continuación, se construye un indicador de la intensidad de facto de la reforma agraria a nivel de comuna (IRA). Teniendo en cuenta que el objetivo principal de la reforma era redistribuir la tierra para aumentar la eficiencia en su uso, se mide la intensidad del proceso como la cantidad de tierra disponible que finalmente fue redistribuida para fines agrarios. En base a esto, el índice que construimos para la intensidad final de la reforma agraria en la comuna $i$ es (el índice a nivel de país es simplemente la suma de los índices comunales):

$I R A_{i}=\frac{\sum_{\mathrm{p} \in \mathrm{i}}(\mathrm{HF} \text { Finalmente Expropiadas del predio } p)-(\text { Transferencias No Agrícolas del predio } p)}{\text { Superficie Total en HF Departamento } i}$

El numerador mide la cantidad de tierra bajo la reforma en la comuna $i$, por lo tanto sumamos todos los segmentos que fueron finalmente expropiados de cada predio $p$ perteneciente a la comuna $i$ neto de las transferencias con fines no agrarios. Transferencias sin objetivos agrarios son aquellas que acabaron en manos de diferentes organizaciones, como la Corporación Nacional Forestal (Conaf), clubes deportivos, municipios o ministerios (por ejemplo, el Ministerio de Educación y el de Bienes Nacionales). Teniendo en cuenta esto, 650.833,3 HF se califican para fines no agrícolas, lo que equivale al 6,63 por ciento de la tierra expropiada inicialmente. En cuanto al denominador, utilizamos la superficie total de la comuna $i$ según el INE (2007). ${ }^{18}$

La elección tanto del numerador como del denominador del IRA lleva supuestos implícitos que creemos razonables. Respecto del de-

${ }^{18}$ En cuanto a la definición de comuna que se utilizó a lo largo del trabajo, se tuvo que lidiar con el problema de que las comunas varían entre 1955 y 2007 y, de hecho, el uso de las definiciones de 2007 conduciría a desajustes y a interpretaciones erróneas. A partir de este punto, lo que hicimos fue construir unidades geográficas que (i) tuviesen características similares y (ii) no cambiasen durante el período de estudio. En algunos casos, ambas condiciones fueron posibles pero en otros fue necesario agrupar hasta 7 comunas para elaborar una unidad que cumpliera tales condiciones. Este procedimiento implica que en lugar de las 346 comunas actuales sólo se utilizaron 271 unidades geográficas, pero haciendo posible comparaciones intertemporales. La información detallada de este proceso se encuentra disponible para quienes lo soliciten. 
nominador, utilizamos la superficie total de la comuna y, por lo tanto, no hacemos diferencia entre las tierras de cultivo y las que no lo son, dado que no existe buena información a nivel de comuna. Respecto del numerador, utilizamos la superficie expropiada medida en HF y no en HRB por dos razones: (i) como mencionamos más arriba, no todas las fichas vienen con HRB; y (ii) no tenemos una buena medida de la superficie total de la comuna en HRB. Sin embargo, es muy probable que la discrepancia entre el numerador y el denominador respecto de medidas más perfectas esté correlacionada con características geográficas y climáticas de la comuna. Por ello, en los ejercicios econométricos reportados más abajo se controla justamente por características de la comuna. Respecto de esto, debe señalarse que no encontramos que estas características expliquen una gran proporción de la variación de IRA en todas las comunas. En consecuencia, creemos que el uso de HF en el numerador y en el denominador del IRA es una buena aproximación para identificar la variación observada en diferentes unidades geográficas del país. ${ }^{19}$

\section{PRINCIPALES HECHOS ESTILIZADOS}

Teniendo en cuenta los predios incluidos en la base de datos, ahora se presenta una serie de indicadores que proporcionan una visión general del proceso de reforma agraria. Es importante destacar que este trabajo no pretende identificar relaciones causales ni resolver interrogantes específicas acerca del proceso de reforma. El objetivo es, a partir de los antecedentes recopilados, la identificación de patrones que revelan tendencias o correlaciones entre cualquiera de los proxies para la intensidad de la reforma y otras variables.

${ }^{19}$ Más aún, existe una alta correlación entre el logaritmo del número de HF y el logaritmo del número de HRB a nivel comunal y a nivel de predio (luego de controlar por efecto comuna). Esto sugiere que si bien se pueden documentar ejemplos individuales (presentes en nuestra base de datos) donde existe una discrepancia entre e intra-comuna entre ambas variables, la correlación promedio es alta, sugiriendo que el uso de HF permite tener una idea adecuada de la variación de reforma agraria entre e intracomunas. Con todo, justamente la disposición de datos a nivel microeconómico permite que investigadores interesados en este punto puedan profundizar en análisis posteriores detallados. 
Figura 2. HISTOGRAMA DE LA SUPERFICIE DE LOS PREDIOS (PREDIOS DE MENOS DE $30.000 \mathrm{HF}$ )

Panel A: En niveles

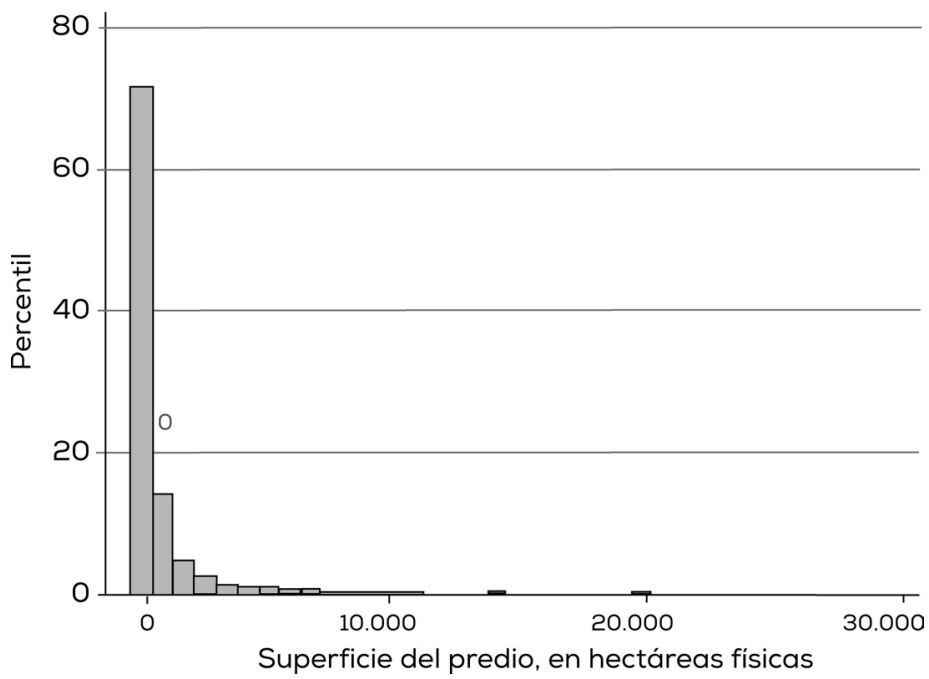

Panel B: En logaritmo natural

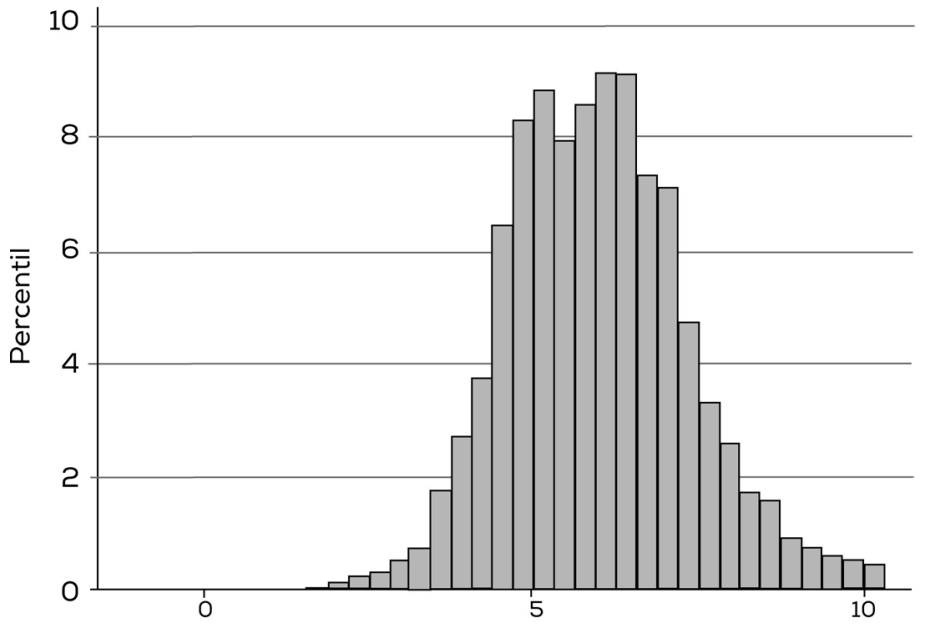

Logaritmo de superficie del predio, en hectáreas físicas 


\subsection{Características de los predios afectados por la reforma}

La figura 2 presenta un histograma de la superficie de los predios que entraron en el proceso. Mientras el panel A muestra la frecuencia para diferentes valores de HF, el panel B muestra la frecuencia para el logaritmo del tamaño de los predios. En ambas figuras excluimos predios con superficie mayor a $30.000 \mathrm{HF}$ (que se supone es un proxy para el tamaño máximo de un predio agrícola utilizado con fines agrarios). ${ }^{20}$ Estas figuras muestran una evidente heterogeneidad en el tamaño de los predios que entraron en el proceso, en el que coexisten predios relativamente pequeños con un conjunto de predios de gran tamaño.

La tabla 2 muestra una serie de hechos estilizados que utilizan la información a nivel de predio. Se presenta información tanto para todos los predios incluidos en la base de datos como también excluyendo predios de más de $30.000 \mathrm{HF}$. Las cifras de la tabla 2 sugieren que la distribución de tamaño de los predios es muy desigual. Mientras que la mediana del tamaño fue de $385 \mathrm{HF}$, el promedio fue de $1.799 \mathrm{HF}$. Cuando se excluyen los predios con un tamaño mayor que $30.000 \mathrm{HF}$ se produce, como era de esperar, una gran disminución en el tamaño medio de los predios a alrededor de $1.132 \mathrm{HF}$ y una pequeña reducción en la mediana a $380 \mathrm{HF}$. Cuando se utilizan HRB y se excluyen los predios con tamaño superior a $30.000 \mathrm{HF}$, las estadísticas no cambian significativamente. Esta similitud es compatible con la conjetura de que los predios muy grandes tenían escaso valor agrícola (como hemos comentado anteriormente, la idea básica de HRB era exactamente corregir HF por diferencias en la productividad).

El hecho de que nuestra base de datos presenta la información a nivel de predios permite examinar la distribución del tamaño de los predios expropiados. Ésta es también una contribución de este trabajo con respecto a la literatura anterior. Presentamos esta información también en la tabla 2 , en la que se presentan los valores correspondientes a los percentiles $1,25,75$ y 99 de la distribución del tamaño de predios

${ }^{20}$ Sólo 55 predios con superficie mayor a $30.000 \mathrm{HF}$ entraron a la reforma, algunos de ellos tan grandes como para tener 200.000 HF. Estos predios con superficie mayor a $30.000 \mathrm{HF}$ representan menos del 1 por ciento de los predios expropiados, pero el 39,2 por ciento del total de tierras expropiadas. Estos predios generalmente no eran adecuados para la producción agraria y algunos de ellos son actualmente reservas nacionales. 
Tabla 2. ESTADÍSTICAS DESCRIPTIVAS DE LOS PREDIOS AFECTADOS POR LA REFORMA AGRARIA

\begin{tabular}{|c|c|c|c|}
\hline & & $\begin{array}{l}\text { Todos los } \\
\text { predios }\end{array}$ & $\begin{array}{l}\text { Terrenos } \\
<30.000 \mathrm{HF}\end{array}$ \\
\hline \multirow{6}{*}{$\begin{array}{l}\text { Estadisticas descriptivas del } \\
\text { tamaño de los predios en HF }\end{array}$} & Promedio & 1.799 & 1.132 \\
\hline & Percentil 1 & 18 & 17 \\
\hline & Percentil 25 & 152 & 150 \\
\hline & Mediana & 385 & 380 \\
\hline & Percentil 75 & 974 & 942 \\
\hline & Percentil 99 & 29.940 & 16.000 \\
\hline \multirow{6}{*}{$\begin{array}{l}\text { Estadísticas descriptivas del } \\
\text { tamaño de los predios en HRB }\end{array}$} & Promedio & 149 & 135 \\
\hline & Percentil 1 & 6 & 6 \\
\hline & Percentil 25 & 59 & 58 \\
\hline & Mediana & 96 & 95 \\
\hline & Percentil 75 & 153 & 150 \\
\hline & Percentil 99 & 1.086 & 703 \\
\hline \multirow{2}{*}{$\begin{array}{l}\text { Predios expropiados al menos } \\
\text { parcialmente }\end{array}$} & Hasta 1973 & $99,7 \%$ & $99,7 \%$ \\
\hline & Al final & $44,2 \%$ & $44,4 \%$ \\
\hline \multirow{2}{*}{$\begin{array}{l}\text { Predios completamente } \\
\text { expropiados }\end{array}$} & Hasta 1973 & $91,4 \%$ & $91,4 \%$ \\
\hline & Al final & $4,2 \%$ & $4,1 \%$ \\
\hline \multirow{2}{*}{$\begin{array}{l}\text { Promedio de la tierra dejada al } \\
\text { antiguo propietario después } \\
\text { de la reforma (en HF) }\end{array}$} & Hasta 1973 & 41.2 & 25,4 \\
\hline & Al final & 169,2 & 161,3 \\
\hline \multirow{2}{*}{$\begin{array}{l}\text { Mediana de la tierra dejada al } \\
\text { antiguo propietario después } \\
\text { de la reforma (en HF) }\end{array}$} & Hasta 1973 & 0 & 0 \\
\hline & Al final & 38 & 39 \\
\hline
\end{tabular}

en HF y HRB. La alta heterogeneidad en el tamaño de los predios se refleja en la distribución, que abarca desde predios muy pequeños (el percentil 1 de la distribución de predios es tan bajo como $18 \mathrm{HF}$ ), hasta predios muy grandes (el percentil 99 de la distribución es tan alto como 29.940 HF). La distribución de tamaño de los predios en términos de HRB (y, por lo tanto, corregido al menos parcialmente por las diferencias en la productividad) es mucho más estrecha, pero persisten grandes 
diferencias en el tamaño del predio: el valor del percentil 99 es más de 150 veces el valor de la percentil 1 de la distribución de tamaño del predio. La figura 3 representa esta heterogeneidad mediante una ampliación de la distribución de predios de tamaño menor que 5.000 HF. De hecho, la información de todos los predios incluidos sugiere que aproximadamente el 75 por ciento de los predios eran de tamaño menor que $1.000 \mathrm{HF}$.

Figura 3. HISTOGRAMA DE SUPERFICIE DE PREDIOS CON SUPERFICIE INFERIOR A $5.000 \mathrm{HF}$

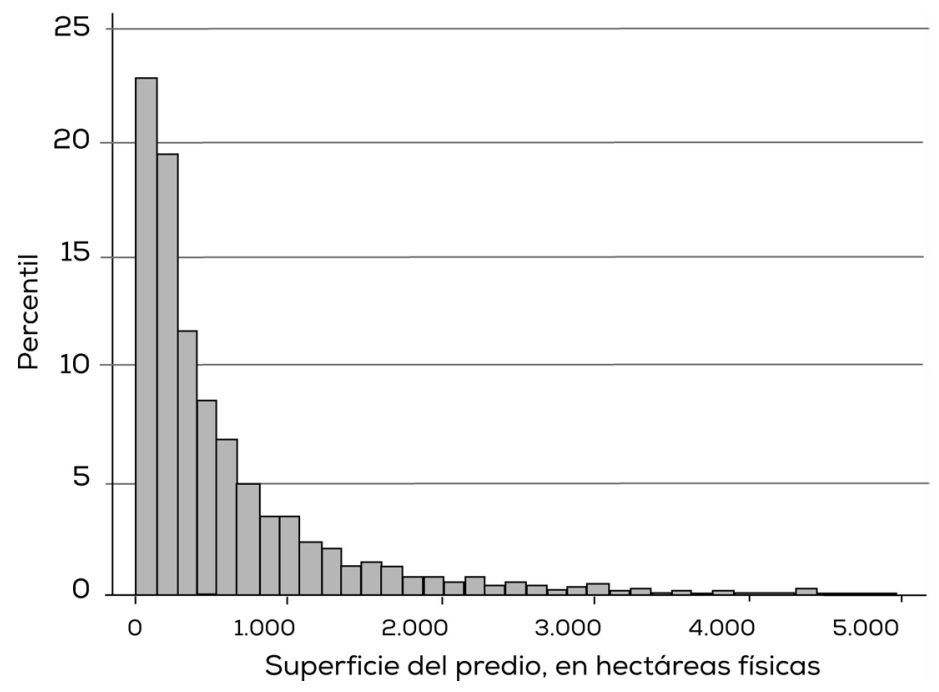

En resumen, los datos sugieren un alto grado de heterogeneidad en el tamaño de los predios expropiados y, por lo tanto, esto implica que no sólo las grandes propiedades entraron en el proceso de reforma, sino que también participaron predios de tamaños muy diferentes: mientras la mediana de la superficie del predio es de $385 \mathrm{HF}$, el percentil 25 es menos de la mitad de la mediana (152 HF) y el percentil 75 es más de dos veces la mediana (974 HF). Esta heterogeneidad no ha sido presentada en investigaciones previas basadas en los archivos de la CORA y muestra el valor de utilizar información a nivel de predios.

Las siguientes filas de la tabla 2 permiten examinar la evolución del proceso distinguiendo lo que sucedió hasta el año 1973 y lo que ocurrió al final de la reforma durante el régimen de Pinochet. Es intere- 
sante notar que hacia 1973 el 91,4 por ciento de los predios había sido completamente expropiado. Esto contrasta con lo que observamos al final del proceso (después de 1973), cuando se aprecia una gran reversión en la intensidad de las expropiaciones. Los datos implican que en ese período sólo 4,2 por ciento de los predios fueron completamente expropiados y gran parte de los predios expropiados inicialmente fueron devueltos a sus propietarios. Volveremos a este punto más adelante.

\subsection{Intensidad de la reforma a nivel comunal}

Pasamos ahora a estudiar la intensidad del proceso de expropiación a nivel comunal. La intensidad promedio del índice IRA a nivel comunal al final del proceso es 19,8 por ciento para todo el país y 21,1 por ciento entre la zona geográfica ubicada entre la Cuarta y la Décima Región, que corresponde a la zona del país más relacionada con la agricultura. A su vez, la mediana del índice IRA a nivel comunal al final del proceso es 11,8 por ciento para todo el país y 13,7 por ciento para la zona más relacionada con la agricultura. Con el fin de estudiar la heterogeneidad de la intensidad del proceso de expropiación dentro del país, la figura 4 presenta el histograma del índice IRA por comuna. Observamos en esta dimensión también un alto grado de heterogeneidad. Mientras que la mayor parte de las observaciones se concentra entre 0 y 20 por ciento, con una desviación estándar de 21,4 por ciento, hay comunas con un grado de intensidad del proceso de expropiación tan alta como 95,4 por ciento. Esto implica que existe heterogeneidad en la intensidad de la expropiación en diferentes comunas, lo que constituye un hecho interesante, que puede tener su explicación en factores endógenos, como la productividad de la tierra, o idiosincráticos, como las características geográficas de la zona o las características de agentes locales de la CORA (a cargo de la aplicación de la reforma). ${ }^{21} \mathrm{~A} 1$ margen de las causas, esto también es un hecho estilizado que no se destaca en la literatura anterior sobre el proceso de reforma agraria chilena y que da cuenta de que en la práctica la intensidad de la reforma varía de modo

${ }^{21}$ Por ejemplo, Garrido (1988) menciona el caso de la muerte de un funcionario de la CORA en la provincia de Linares mientras tomaba el control de una propiedad en 1970. La reacción a este suceso fue un aumento en la velocidad del proceso de expropiación en esa provincia. 
Figura 4. HISTOGRAMA DE LA INTENSIDAD DE LA REFORMA AGRARIA A NIVEL COMUNAL

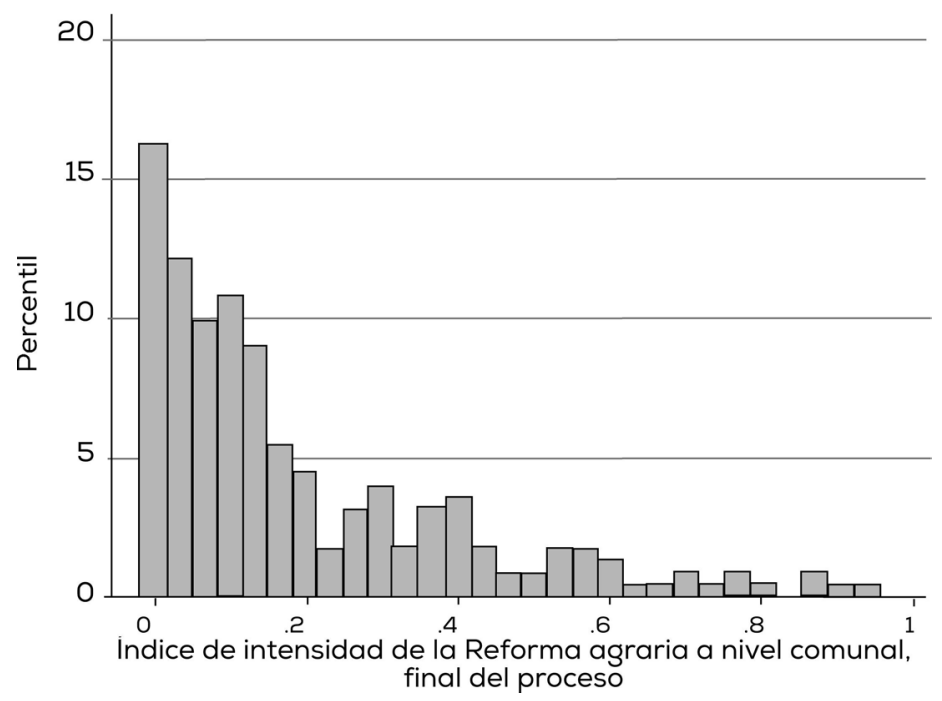

importante en diferentes zonas del país. La investigación futura podría tratar de usar esta heterogeneidad a nivel de comuna para explicar otros resultados, tales como las diferencias en la productividad posterior en diferentes comunas.

\subsection{Los tiempos de la reforma}

Para entender el proceso de expropiaciones de una mejor manera, a continuación exploramos su evolución temporal. Considerando la investigación previa sobre el tema, dividimos el período cubierto por la base de datos en las siguientes etapas:

- Hasta 1961. Este período involucra expropiaciones hasta que la Ley 15.020 fue promulgada bajo el gobierno de Alessandri.

-1962-1964. En este período se aplica la reforma bajo el gobierno de Alessandri.

- 1964-1967. En esta etapa la reforma avanza en el gobierno de Frei antes de la aprobación de la Ley 16.640.

-1967-1970. Este período corresponde a la reforma en el gobierno de Frei tras la aprobación de la Ley 16.640. 
Tabla 3. EVOLUCIÓN DE LAS EXPROPIACIONES

\begin{tabular}{lccccc}
\hline & $1962-1964$ & $1964-1967$ & $1967-1970$ & $1970-1973$ & $1973-1980$ \\
\hline $\begin{array}{l}\text { Predios } \\
\text { expropiados }\end{array}$ & 17 & 418 & 875 & 4.212 & 142 \\
\hline $\begin{array}{l}\text { Porcentaje del } \\
\text { total de predios } \\
\text { expropiados }\end{array}$ & $0,30 \%$ & $7,38 \%$ & $15,45 \%$ & $74,36 \%$ & $2,51 \%$ \\
\hline HF expropiadas & $111.827,8$ & $1.034 .650,0$ & $2.519 .604,0$ & $6.012 .740,0$ & $224.923,1$ \\
\hline $\begin{array}{l}\text { Porcentaje del } \\
\text { total de HF } \\
\text { expropiadas }\end{array}$ & $1,13 \%$ & $10,45 \%$ & $25,44 \%$ & $60,71 \%$ & $2,27 \%$ \\
\hline $\begin{array}{l}\text { Promedio HF } \\
\text { Mediana HF }\end{array}$ & $6.578,11$ & $2.475,24$ & $2.879,55$ & $1.427,53$ & $1.583,97$ \\
\hline
\end{tabular}

-1970-1973. Este período corresponde a la aplicación de la reforma bajo el gobierno de Allende usando la Ley 16.640.

-1973-1980. Abarca el período de contrarreforma parcial.

A partir de 1980. Corresponde a los ajustes restantes de la reforma.

La tabla 3 muestra que efectivamente el período 1970-1973 fue la etapa cuando se verifica la mayor expropiación de predios y de superficie. ${ }^{22}$ Los datos sugieren, además, que mientras en 1962-1969 estaban bajo amenaza de expropiación los grandes predios, en el período 19701973 esto cambió y predios de tamaño mucho menor también quedaron en riesgo de expropiación.

La evolución de la intensidad de la reforma es una forma alternativa de entender el proceso de reforma agraria en sí y también una forma de identificar diferencias entre las unidades geográficas, ya sean comunas o regiones. La tabla 4 presenta la evolución de nuestro índice IRA por región, así como su media, mediana y desviación estándar. ${ }^{23} \mathrm{~A}$ su vez, la última fila de esta tabla presenta la intensidad de la reforma a nivel del país (o sea, considerando en el numerador todos los predios expropiados y en el denominador la superficie total del país). Los datos confirman que el clímax de la reforma fue durante el gobierno de Allende, que es cuando el índice IRA alcanza su máximo para todas las

${ }^{22}$ Los periodos anteriores a 1961 y después de 1980 se omitieron en la tabla, pues no son importantes para la comprensión de la reforma, y debido a que la magnitud de los acontecimientos en esos períodos los hace irrelevantes.

${ }^{23} \mathrm{El}$ anexo presenta una descripción de las unidades geográficas utilizadas en este trabajo. 
Tabla 4. ÍNDICE DE INTENSIDAD DE LA REFORMA AGRARIA (IRA) A TRAVÉS DEL TIEMPO Y INTENSIDAD DE LA CONTRAREFORMA (\%)

\begin{tabular}{lrrrrrrrrrr}
\hline Región & 1962 & 1964 & 1967 & 1970 & 1973 & 1975 & 1980 & 1990 & 2001 & $\begin{array}{c}\text { Contra- } \\
\text { rreforma }\end{array}$ \\
\hline I & 0,21 & 0,21 & 0,74 & 0,78 & 0,78 & 0,78 & 0,80 & 0,79 & 0,79 & $-1,3 \%$ \\
II & 0,00 & 0,00 & 0,00 & 0,00 & 0,06 & 0,05 & 0,04 & 0,04 & 0,04 & $26,2 \%$ \\
III & 0,00 & 0,00 & 0,00 & 0,00 & 0,92 & 0,43 & 0,38 & 0,38 & 0,38 & $59,1 \%$ \\
IV & 0,50 & 3,14 & 10,20 & 21,29 & 35,94 & 34,47 & 29,74 & 29,47 & 29,47 & $18,0 \%$ \\
V & 0,00 & 0,00 & 11,10 & 36,12 & 58,84 & 55,00 & 51,93 & 52,13 & 52,13 & $11,4 \%$ \\
VI & 0,00 & 0,00 & 2,56 & 7,63 & 45,54 & 37,30 & 33,10 & 28,69 & 28,65 & $37,1 \%$ \\
VII & 0,44 & 0,49 & 1,53 & 5,85 & 28,11 & 16,80 & 14,64 & 12,53 & 11,69 & $58,4 \%$ \\
VIII & 0,15 & 0,51 & 1,42 & 3,10 & 27,86 & 26,17 & 16,83 & 14,03 & 14,03 & $49,6 \%$ \\
IX & 0,11 & 0,15 & 0,99 & 3,13 & 21,68 & 13,55 & 8,00 & 7,84 & 7,84 & $63,9 \%$ \\
X & 0,00 & 0,00 & 0,10 & 5,48 & 26,93 & 20,38 & 15,50 & 14,48 & 14,33 & $46,8 \%$ \\
XI & 0,00 & 0,44 & 1,12 & 1,83 & 3,15 & 3,68 & 3,57 & 3,56 & 3,46 & $-10,0 \%$ \\
XII & 0,00 & 0,00 & 0,71 & 3,04 & 13,18 & 12,64 & 12,07 & 12,00 & 12,00 & $9,00 \%$ \\
RM & 0,02 & 0,02 & 14,70 & 20,63 & 61,99 & 36,93 & 22,73 & 19,96 & 19,94 & $67,8 \%$ \\
\hline Media & 0,11 & 0,38 & 3,48 & 8,37 & 25,00 & 19,86 & 16,10 & 15,07 & 14,98 & $33,5 \%$ \\
Mediana & 0,00 & 0,02 & 1,12 & 3,13 & 26,93 & 16,80 & 14,64 & 12,53 & 12,00 & $37,1 \%$ \\
Std. & 0,18 & 0,85 & 5,00 & 10,90 & 21,40 & 17,28 & 15,23 & 14,83 & 14,84 & $26,3 \%$ \\
Dev. & & & & & & & & & & \\
\hline Pais & 0,15 & 0,29 & 1,94 & 5,43 & 29,20 & 21,88 & 15,88 & 14,12 & 13,97 & $52,2 \%$ \\
\hline
\end{tabular}

Notas: Las primeras 9 columnas presentan promedios regionales del indice de intensidad de la reforma agraria (IRA) y la décima columna presenta la intensidad de la contrarreforma que se define como: (IRA $1973-$ IRA $\left._{2001}\right) /$ IRA $_{1973}$

regiones excepto en la Primera y Undécima. ${ }^{24}$ En cuanto a las diferencias entre regiones, como se esperaba, la zona central (es decir, aquella que va entre la Cuarta y la Décima Región) concentró la mayoría de las expropiaciones.

Otro aspecto destacable es que existen claras diferencias en la intensidad del proceso de contrarreforma a nivel regional, como muestra

${ }^{24}$ La mayoría de las comunas de estas regiones carecen de tierra de uso agrícola, por lo que la evolución de la intensidad de la reforma puede ser bastante irrelevante. 
la última columna de la tabla 4. La intensidad del proceso de contrarreforma es simplemente el cambio porcentual en la intensidad de la reforma entre 1973 y 2001. Así, éste es un indicador de la cantidad de expropiaciones originales que se deshicieron.

Los resultados de la última columna de la tabla 4 implican que hubo gran heterogeneidad en esta dimensión también. Por ejemplo, las regiones Tercera, Séptima, Novena y Metropolitana tuvieron una fuerte contrarreforma, que deshizo el 60 por ciento o más de las expropiaciones iniciales de tierra. O sea, sólo el 40 por ciento de la tierra que entró en el proceso finalmente fue expropiada (parte de ella se distribuyó entre pequeños propietarios y parte de ella fue subastada, como veremos más adelante). En contraste, otras regiones - como la Primera, Cuarta, Quinta, Undécima y Duodécima - tienen un proceso de contrarreforma mucho más modesto. Esta heterogeneidad, que es apoyada por una alta desviación estándar $(0,26)$, no tiene una explicación obvia. Puede estar relacionada con atributos particulares del proceso regional de contrarreforma en sí, o con un proceso de reforma regional excesivo que pudo justificar una contrarreforma más fuerte. Esto refuerza el punto mencionado anteriormente respecto de la alta heterogeneidad que se observa en la reforma agraria en diferentes zonas del país. Una vez más, éste es un punto que no se discute en las descripciones de la reforma anteriores a este artículo e invita a investigación adicional en el futuro.

\subsection{Las razones legales de la reforma}

Los argumentos jurídicos usados para la reforma y presentes en la base de datos son las razones $1,2,3,4,5,6,8,10$ y 15 (ver tabla 1 para la lista de todas las causales). La tabla 5 muestra que las razones más relevantes para el período completo fueron la causal 3 (es decir, predios mayores que $80 \mathrm{HRB}$ ), la causal 4 (predios que estaban explotados ineficientemente o abandonados), la causal 6 (predios cuyos propietarios son personas jurídicas de derecho público o privado) y la causal 10 (predios ofrecidos por sus propietarios para la adquisición por la CORA).

La tabla 5 también presenta la evolución en el uso de las diferentes razones legales en diferentes períodos: el gobierno de Frei antes de 1967, el gobierno de Frei entre 1967 y 1970, y el gobierno de Allende entre 1970 y 1973. Se observa que la razón 3 (predio agrícola demasiado grande) aumentó su importancia a través del tiempo y terminó 
Tabla 5. RAZONES DE EXPROPIACIÓN POR PERÍODO

\begin{tabular}{c|cccc|cccc}
\hline \multicolumn{3}{c}{ Panel A: Predios } & \multicolumn{5}{c}{ Panel B: Superficie (HF) } \\
\hline $\begin{array}{c}\text { Razón } \\
\text { Legal }\end{array}$ & $\begin{array}{c}\text { Periodo } \\
\text { completo }\end{array}$ & $1964-$ & $1967-$ & $1970-$ & Periodo & $1964-$ & $1967-$ & $1970-$ \\
\hline 1 & 84 & 2 & 82 & 0 & 15.059 & 1.268 & 13.791 & 0 \\
& $1,6 \%$ & $2,1 \%$ & $9,8 \%$ & $0,0 \%$ & $0,2 \%$ & $0,4 \%$ & $0,7 \%$ & $0,0 \%$ \\
\hline 2 & 9 & 3 & 0 & 6 & 9.120 & 6.009 & 0 & 3.111 \\
& $0.2 \%$ & $3,2 \%$ & $0,0 \%$ & $0,1 \%$ & $0,1 \%$ & $2,0 \%$ & $0,0 \%$ & $0,1 \%$ \\
\hline 3 & 2.225 & 2 & 306 & 1.917 & 3.180 .321 & 310 & 637.663 & 2.542 .348 \\
& $43,7 \%$ & $2,1 \%$ & $36,4 \%$ & $46,1 \%$ & $38,6 \%$ & $0,1 \%$ & $31,7 \%$ & $42,9 \%$ \\
\hline 4 & 930 & 1 & 11 & 918 & 658.054 & 1.040 & 8.231 & 648.783 \\
& $18,3 \%$ & $1,1 \%$ & $1,3 \%$ & $22,1 \%$ & $8,0 \%$ & $0,3 \%$ & $0,4 \%$ & $10,9 \%$ \\
\hline 5 & 30 & 1 & 22 & 7 & 16.883 & 125 & 2.561 & 14.197 \\
& $0,6 \%$ & $1,1 \%$ & $2,6 \%$ & $0,2 \%$ & $0,2 \%$ & $0,0 \%$ & $0,1 \%$ & $0,2 \%$ \\
\hline 6 & 355 & 1 & 63 & 291 & 2.425 .961 & 415 & 584.141 & 1.841 .405 \\
& $7,0 \%$ & $1,1 \%$ & $7,5 \%$ & $7,0 \%$ & $29,4 \%$ & $0,1 \%$ & $29,1 \%$ & $31,0 \%$ \\
\hline 8 & 89 & 0 & 0 & 89 & 160.701 & 0 & 0 & 160.701 \\
& $1,7 \%$ & $0,0 \%$ & $0,0 \%$ & $2,1 \%$ & $2,0 \%$ & $0,0 \%$ & $0,0 \%$ & $2,7 \%$ \\
\hline 10 & 1.291 & 7 & 354 & 930 & 1.486 .676 & 3.876 & 761.521 & 721.279 \\
& $25,3 \%$ & $7,4 \%$ & $42,1 \%$ & $22,4 \%$ & $18,0 \%$ & $1,3 \%$ & $37,9 \%$ & $12,2 \%$ \\
\hline 15 & 81 & 78 & 2 & 1 & 287.801 & 285.727 & 1.284 & 791 \\
& $1,6 \%$ & $82,1 \%$ & $0,2 \%$ & $0,0 \%$ & $3,5 \%$ & $95,6 \%$ & $0,1 \%$ & $0,0 \%$ \\
\hline Total & 5.094 & 95 & 840 & 4.159 & 8.240 .578 & 298.771 & 2.009 .191 & 5.932 .616 \\
\hline \multirow{2}{*}{5}
\end{tabular}

siendo la razón más importante para expropiar, con más del 40 por ciento de las expropiaciones en el último período. Además, la causal 4 (explotación ineficiente del predio agrícola) alcanzó una gran importancia en el período de Allende, mientras que la razón 10 (predio agrícola ofrecido por el propietario) alcanzó relevancia en el período 1967-1970. Esto último refleja probablemente un efecto de expectativas en que los propietarios de la tierra supusieron que la expropiación se iba a producir de todos modos. ${ }^{25}$

${ }^{25}$ La información disponible en la base de datos también permite estudiar qué razones dominaron durante la reforma en cada comuna. Por razones de espacio, no reportamos un análisis detallado, pero la información disponible permite apreciar que existe alta heterogeneidad en esta dimensión, consistente con los resultados previos. 


\subsection{Intensidad de la reforma: correlaciones}

Un desafío interesante es comprender lo que realmente motivó la reforma agraria, más allá de lo establecido legalmente. Tratamos de estudiar esta cuestión a través de una serie de ejercicios que intentan proporcionar evidencia sobre las razones de la heterogeneidad observada en la intensidad de la reforma agraria en todas las comunas. Ya que la reforma de Alessandri era mucho más modesta que la de Frei y Allende la omitimos de este análisis, y nos concentramos en cómo Frei y Allende realizaron respectivamente sus procesos de reforma. Además, separamos el periodo de Frei en dos para este análisis, distinguiendo dos etapas — 1964-1967 y 1967-1970 — , pues la Ley 16.640 puede haber cambiado las motivaciones de la reforma.

Con este objetivo, se estiman regresiones de la intensidad de la reforma a nivel de comuna contra características económicas, sociales y geográficas observadas antes de que comenzara la reforma. En primer lugar, estimamos regresiones de la intensidad de la reforma a nivel comunal durante los tres periodos indicados anteriormente respecto a variables binarias (dummies) por región. ${ }^{26}$ La tabla 6 muestra los resultados: estas regresiones explican 8,9 por ciento, 19,5 por ciento y 21,2 por ciento de la variación en la intensidad de la reforma agraria. A continuación, en las siguientes tres columnas de la tabla 6 , se estudia qué sucede al añadir variables geográficas, como la media y la varianza de temperatura y precipitaciones. La motivación para incluir estas variables es doble. En primer lugar, como discutimos más arriba, estas variables buscar capturar potencial variación entre comunas en la calidad de la tierra (recordar que el índice IRA supone que el uso de HF en el numerador y denominador provee una aproximación razonable). En segundo lugar, los trabajos empíricos que utilizan variación comunal o regional incorporan este tipo de variables para capturar potenciales efectos geográficos sobre las variables de interés (ver, por ejemplo, Cuesta et al. 2015).

Los resultados presentados en las columnas 4 a 6 sugieren que casi ninguna de estas variables tiene efectos estadísticamente significativos y no agregan mucho a la variación de la intensidad de la reforma ex-

${ }^{26}$ En todas estas regresiones sólo incorporamos comunas ubicadas entre la Cuarta y Décima Región, es decir, el área en las que la agricultura era relevante. 
Tabla 6. REGRESIONES DE LA INTENSIDAD DE LA REFORMA AGRARIA (IRA) SOBRE EFECTO FIJOS Y CONTROLES GEOGRÁFICOS

\begin{tabular}{|c|c|c|c|c|c|c|}
\hline & $\begin{array}{c}\text { (1) } \\
\text { Reforma } \\
\text { en } 1967\end{array}$ & $\begin{array}{c}\text { (2) } \\
\text { Reforma } \\
\text { en } 1970\end{array}$ & $\begin{array}{c}\text { (3) } \\
\text { Reforma } \\
\text { en } 1973\end{array}$ & $\begin{array}{c}\text { (4) } \\
\text { Reforma } \\
\text { en } 1967\end{array}$ & $\begin{array}{c}\text { (5) } \\
\text { Reforma } \\
\text { en } 1970\end{array}$ & $\begin{array}{c}\text { (6) } \\
\text { Reforma } \\
\text { en } 1973\end{array}$ \\
\hline \multirow[t]{2}{*}{ Región 4} & 0.0142 & 0.0427 & -0.136 & 0.0450 & 0.0421 & 0.0188 \\
\hline & (0.0383) & $(0.0594)$ & (0.0838) & $(0.0635)$ & (0.0989) & (0.139) \\
\hline \multirow[t]{2}{*}{ Región 5} & 0.0465 & $0.104^{*}$ & -0.0350 & 0.0500 & 0.0963 & 0.0377 \\
\hline & $(0.0375)$ & $(0.0583)$ & (0.0822) & $(0.0441)$ & (0.0688) & $(0.0967)$ \\
\hline \multirow[t]{2}{*}{ Región 6} & -0.0180 & -0.0665 & -0.0632 & -0.0433 & -0.0794 & -0.0996 \\
\hline & $(0.0327)$ & $(0.0507)$ & $(0.0715)$ & $(0.0400)$ & $(0.0624)$ & $(0.0877)$ \\
\hline \multirow[t]{2}{*}{ Región 7} & -0.0373 & $-0.0918^{*}$ & $-0.190^{* \star *}$ & $-0.0693^{*}$ & $-0.109^{*}$ & $-0.212^{* *}$ \\
\hline & (0.0329) & $(0.0511)$ & $(0.0721)$ & $(0.0410)$ & (0.0639) & (0.0898) \\
\hline \multirow[t]{2}{*}{ Región 8} & -0.0485 & $-0.140^{* * *}$ & $-0.304^{* * *}$ & $-0.110^{\star *}$ & $-0.183^{* \star}$ & $-0.325^{\star * *}$ \\
\hline & $(0.0310)$ & $(0.0481)$ & $(0.0679)$ & $(0.0499)$ & $(0.0777)$ & (0.109) \\
\hline \multirow[t]{2}{*}{ Región 9} & -0.0563 & $-0.143^{* * *}$ & $-0.310^{* * *}$ & $-0.138^{* *}$ & $-0.218^{\star *}$ & $-0.333^{* * *}$ \\
\hline & $(0.0341)$ & $(0.0530)$ & $(0.0747)$ & $(0.0581)$ & $(0.0906)$ & (0.127) \\
\hline \multirow[t]{2}{*}{ Región 10} & $-0.0656^{\star *}$ & $-0.132^{\star \star \star}$ & $-0.291^{\star \star \star}$ & $-0.167^{\star \star}$ & $-0.239^{\star *}$ & $-0.286^{* *}$ \\
\hline & $(0.0327)$ & $(0.0507)$ & $(0.0715)$ & $(0.0652)$ & $(0.102)$ & $(0.143)$ \\
\hline log(Promedio & & & & 0.248 & 0.197 & 0.253 \\
\hline lluvi & & & & $(0.247)$ & $(0.3$ & (0 \\
\hline $\begin{array}{l}\text { log(Promedio } \\
\text { temperatura } \\
\text { anual) }\end{array}$ & & & & $\begin{array}{l}0.0302 \\
(0.162)\end{array}$ & $\begin{array}{l}-0.0287 \\
(0.252)\end{array}$ & $\begin{array}{l}-0.158 \\
(0.354)\end{array}$ \\
\hline $\begin{array}{l}\text { log(Varianza } \\
\text { lluvias anuales) }\end{array}$ & & & & $\begin{array}{l}-0.103 \\
(0.122)\end{array}$ & $\begin{array}{c}-0.0855 \\
(0.190)\end{array}$ & $\begin{array}{l}-0.115 \\
(0.267)\end{array}$ \\
\hline $\begin{array}{l}\text { log(Varianza } \\
\text { temperatura } \\
\text { anual) }\end{array}$ & & & & $\begin{array}{l}-0.0246 \\
(0.0260)\end{array}$ & $\begin{array}{l}-0.0475 \\
(0.0406)\end{array}$ & $\begin{array}{c}0.105^{*} \\
(0.0570)\end{array}$ \\
\hline \multirow[t]{2}{*}{ Constante } & $0.0665^{\star \star \star}$ & $0.178^{* * *}$ & $0.540^{* * *}$ & -0.352 & 0.141 & 0.143 \\
\hline & (0.0253) & $(0.0393)$ & $(0.0554)$ & $(0.543)$ & $(0.847)$ & $(1.191)$ \\
\hline Observaciones & 185 & 185 & 185 & 185 & 185 & 185 \\
\hline $\mathrm{R} 2$ & 0.089 & 0.186 & 0.206 & 0.108 & 0.198 & 0.223 \\
\hline
\end{tabular}

La Región Metropolitana es la categoría omitida entre los efectos fijos por región. Error estándar entre paréntesis. ${ }^{* \star *} p<0.01,{ }^{* *} p<0.05,{ }^{*} p<0.1$ 
plicada por el modelo. Estos resultados sugieren que la heterogeneidad observada entre diferentes comunas del país puede ser explicada de modo sólo parcial por diferentes características de las comunas, que presumiblemente están correlacionadas con aspectos relacionados a las características de la tierra, y con ello se validan, al menos parcialmente, los supuestos utilizados en la construcción del índice IRA.

A continuación expandimos este análisis para estudiar si aspectos relacionados con la eficiencia y la estructura de la propiedad de la tierra. En la tabla 7 se examina la correlación entre la intensidad de la reforma a nivel comunal y dos medidas de eficiencia de la tierra: la producción por hectárea y la producción por trabajador (luego de controlar por las variables incorporadas en la tabla 6 , o sea, variables que identifican la región a la que pertenece la comuna y las variables geográficas). ${ }^{27}$ Los resultados sugieren que a pesar de que en todos los casos el coeficiente estimado es positivo, casi ninguno es estadísticamente significativo, lo que puede descartarlos al menos parcialmente como un factor relevante de la reforma. La única excepción es la correlación entre la producción por trabajador en 1965 y la intensidad de la reforma, que presenta un coeficiente positivo y estadísticamente significativo. Estos resultados contradicen algunas de las descripciones previas de la reforma, pues sugieren que la intensidad de la reforma fue más fuerte en aquellas zonas que eran más productivas en 1965. En otras palabras, estas correlaciones sugieren que es poco probable que la motivación en la práctica de la reforma tuviese que ver con la expropiación en zonas con baja productividad, lo que es consistente con las razones invocadas para las expropiaciones descritas anteriormente (donde la razón que invoca un uso ineficiente de la tierra representa menos del 10 por ciento de la superficie expropiada) y con el hecho que discutimos en la sección 4.6, respecto a que una parte importante de las expropiaciones realizadas invocando ineficiencia en la producción fueron revocadas posteriormente en la contrarreforma (ver tabla 9).

${ }^{27}$ La medida de producción a nivel comunal fue construida por Cuesta et al. (2015). Este trabajo utiliza las medidas presentadas para 1965 (antes de la reforma). Los autores usan información de producción, superficie explotada y trabajadores para un subconjunto de productos que incluyen los siguientes sectores: forestal, frutícola, ganadería y productos primarios. 
Tabla 7. REGRESIONES DE LA INTENSIDAD DE LA REFORMA AGRARIA (IRA) A NIVEL COMUNAL Y MEDIDAS DE EFICIENCIA

\begin{tabular}{lcccccc}
\hline & $\begin{array}{c}(1) \\
\text { Reforma } \\
\text { en 1967 }\end{array}$ & $\begin{array}{c}(2) \\
\text { Reforma } \\
\text { en 1970 }\end{array}$ & $\begin{array}{c}(3) \\
\text { Reforma } \\
\text { en 1973 }\end{array}$ & $\begin{array}{c}(4) \\
\text { Reforma } \\
\text { en 1967 }\end{array}$ & $\begin{array}{c}(5) \\
\text { Reforma } \\
\text { en 1970 }\end{array}$ & $\begin{array}{c}(6) \\
\text { Reforma } \\
\text { en 1973 }\end{array}$ \\
\hline $\begin{array}{l}\text { Log (Producto } \\
\text { por hectárea }\end{array}$ & $\begin{array}{l}0.0308 \\
(0.0216)\end{array}$ & $\begin{array}{l}0.0464 \\
(0.0337)\end{array}$ & $\begin{array}{c}0.0124 \\
(0.0476)\end{array}$ & & & \\
1965) & {$[0.5048]$} & & {$[0.3714]$} & & & \\
Log (Producto & & & & 0.00539 & 0.0118 & $0.0583^{* *}$ \\
por trabajador & & & & $(0.0132)$ & $(0.0205)$ & $(0.0285)$ \\
$1965)$ & & & & $0.6504]$ & & {$[0.0395]$} \\
\hline R2 & 0.119 & 0.207 & 0.223 & 0.109 & 0.200 & 0.241 \\
\hline
\end{tabular}

Todas las regresiones emplean 185 observaciones, incluyen controles geográficos y efectos fijos por región. La Región Metropolitana es la categoría omitida.

Valor-p de test de igualdad de coeficientes en [ ].

Error estándar entre paréntesis. ${ }^{* \star *} p<0.01,{ }^{* *} p<0.05,{ }^{*} p<0$.

La tabla 8 presenta regresiones en las que se estudia la correlación entre la intensidad de la reforma a nivel comunal y variables que miden la estructura de la propiedad de la tierra, a saber, el Gini de la distribución de tierra por comuna, la fracción de tierras ocupadas por predios de tamaño mayor que $2.000 \mathrm{HF}$, y el tamaño medio de los predios. Los resultados sugieren que el Gini de la distribución de la tierra siempre se correlaciona significativamente con la intensidad de la reforma. De hecho, los coeficientes aumentan a medida que pasa el tiempo, lo que implica que el Gini está más correlacionado con la intensidad de la reforma al final del proceso. Verificamos esta diferencia con una prueba estadística de la igualdad de los coeficientes entre las distintas etapas del proceso de reforma, encontrando que efectivamente la desigualdad inicial en la tenencia de la tierra explica efectiva y crecientemente la heterogeneidad de la reforma entre comunas.

Los coeficientes correspondientes a la fracción de la tierra ocupada por predios de tamaño mayor que $2.000 \mathrm{HF}$ y al tamaño medio de los predios sólo son significativamente positivos para el período de Allende. Esto sugiere que esa fase de la reforma estuvo más influenciada por los aspectos de tenencia de la tierra que las etapas previas. 
En conjunto, estos tres resultados indican que los problemas de desigualdad y de tenencia de la tierra parecen haber sido motivaciones en la práctica de la reforma agraria. Es interesante notar que detrás de la correlación de la estructura de la tierra con la intensidad de la reforma está tanto la fracción de la propiedad correspondiente a predios grandes, como la desigualdad de estructura de propiedad. Los mecanismos que pueden explicar la influencia de ambos márgenes son diferentes.

Por ejemplo, en el caso de la desigualdad (medida por el índice Gini) pueden existir tanto una motivación de "eficiencia" (donde zonas con mayor desigualdad tienen mayores diferencias en escala de producción y por ello requerirían mayor intensidad de la reforma), como argumentos de economía política, en que zonas con mayor desigualdad pueden gatillar mayor intensidad expropiatoria en la práctica. Futuros trabajos pueden adentrarse en una discusión más detallada que busque distinguir ambos tipos de motivaciones (probablemente usando variación exógena para identificar relaciones causales). Con todo, parece interesante el aumento de la influencia de factores de la propiedad de la tierra a través de los períodos analizados, lo que sugiere que la re-

Tabla 8. REGRESIÓN DE LA INTENSIDAD DE LA REFORMA AGRARIA (IRA) A NIVEL COMUNAL Y MEDIDAS DE DESIGUALDAD EN LA TENENCIA DE LA TIERRA

\begin{tabular}{|c|c|c|c|c|c|c|}
\hline & \multicolumn{2}{|c|}{ Gini tierras 1965} & \multicolumn{2}{|c|}{$\begin{array}{c}\text { Fracción de tierras en } \\
\text { predios > } 2.000 \mathrm{HF} \\
\text { en } 1965\end{array}$} & \multicolumn{2}{|c|}{$\begin{array}{l}\text { Log(tamaño medio de } \\
\text { predios en 1965) }\end{array}$} \\
\hline & Coef & $\mathrm{R} 2$ & Coef & $\mathrm{R} 2$ & Coef & $\mathrm{R} 2$ \\
\hline $\begin{array}{l}\text { Reforma } \\
\text { en } 1967\end{array}$ & $\begin{array}{c}0.821^{*} \\
(0.441 \\
{[0.0033]}\end{array}$ & 0.126 & $\begin{array}{l}0.00539 \\
(0.0132) \\
{[0.0118]}\end{array}$ & 0.113 & $\begin{array}{c}0.00718 \\
(0.00986) \\
{[0.3038]}\end{array}$ & 0.111 \\
\hline $\begin{array}{l}\text { Reforma } \\
\text { en } 1970\end{array}$ & $\begin{array}{c}2.206^{* * *} \\
(0.673) \\
{[0.0000]}\end{array}$ & 0.245 & $\begin{array}{c}0.0118 \\
(0.0205) \\
{[0.0086]}\end{array}$ & 0.198 & $\begin{array}{c}0.0181 \\
(0.0153) \\
{[0.0003]}\end{array}$ & 0.205 \\
\hline $\begin{array}{l}\text { Reforma } \\
\text { en } 1973\end{array}$ & $\begin{array}{c}5.672^{* * *} \\
(0.874)\end{array}$ & 0.376 & $\begin{array}{l}0.0583^{* *} \\
(0.0285)\end{array}$ & 0.260 & $\begin{array}{l}0.0794^{* * *} \\
(0.0208)\end{array}$ & 0.283 \\
\hline
\end{tabular}

Todas las regresiones emplean 185 observaciones, incluyen controles geográficos y efectos fijos por región. La Región 13 es la categoría omitida.

P-valor de test de igualdad de coeficientes en [ ].

Error estándar entre paréntesis. ${ }^{* * *} p<0.01,{ }^{* *} p<0.05,{ }^{*} p<0$. 
levancia de estos factores como causa de la reforma aumentó a través del tiempo, haciendo que la reforma fuese cada vez más un fenómeno endógeno. ${ }^{28}$

\subsection{La contrarreforma}

Como ya hemos explicado, la reforma agraria aumentó en intensidad a medida que pasaba el tiempo o, como Bellisario (2007a; 2007b) sostiene, se distanció de su motivación legal original. La intensidad más alta tuvo lugar durante el gobierno de Allende, que duró hasta 1973 cuando los militares tomaron el poder. La política principal en la etapa siguiente fue detener la reforma y regularizar la situación de los predios expropiados (la mayoría de los cuales no tenían propietarios y fueron realmente administradas por el gobierno como las empresas estatales). El gobierno militar utilizó para ello los siguientes mecanismos:

1. Revocación de expropiaciones o renegociación cuando el propietario original sostenía que el proceso fue ilegal.

2. Distribución de propiedad entre campesinos que habían sido beneficiados originalmente por la reforma.

3. Transferencia de tierras expropiadas a instituciones (generalmente estatales).

4. Subasta a la mejor oferta.

5. Devolución a los antiguos propietarios.

La tabla 9 presenta cálculos de la frecuencia de cada uno de estos posibles resultados para los predios reformados, utilizando el número observado de casos, y luego ponderando por el tamaño de cada predio. La distribución entre campesinos de la tierra seguida por la revocación son los resultados más frecuentes obtenidos usando ambas formas de medición. El empleo de estadísticas ponderadas por el tamaño de los predios no altera las principales conclusiones, sólo hace que la distribución de la propiedad sea menos significativa y aumente la importancia de la

28 Además de los análisis presentados en esta sección, también estimamos regresiones que incluyen tanto medidas de eficiencia como de estructura de la propiedad de la tierra. Los resultados obtenidos por separado en las regresiones presentadas en las tablas 7 y 8 se mantienen cuando se incorporan todas las variables en una regresión. Sumado a eso, no se observa que existan interacciones entre motivos de eficiencia y de estructura de la propiedad. No reportamos estos resultados para ahorrar espacio. 
Tabla 9. RESULTADOS DE LOS PREDIOS EXPROPIADOS (\%)

\begin{tabular}{lcc}
\hline Canal & No ponderado & Ponderado por tamaño \\
\hline Distribuido & 65.81 & 51.29 \\
Revocado & 29.32 & 35.6 \\
Transferido & 2.05 & 8.25 \\
Subastado & 2.05 & 4.49 \\
Restaurado & 0,50 & 0,06 \\
Otro & 0,27 & 0,29 \\
\hline
\end{tabular}

revocación, las transferencias y las subastas. Esto implica que los propietarios originales que al inicio de la reforma tenían predios más grandes se esforzaron en recuperarlos durante la contrarreforma. Además, el aumento de la participación de las transferencias es apoyado por la entrega de una cantidad considerable de grandes predios a instituciones estatales, principalmente a la Corporación Nacional Forestal (Conaf), alcanzando un tamaño medio de transferencia de 1.469,47 HF. Ambos indicadores respaldan que la reforma dirigida por Alessandri, Frei y Allende fue disuelta, al menos parcialmente, por el gobierno militar.

En cuanto al desarrollo de la contrarreforma, exploramos la relación entre las razones legales invocadas para las expropiaciones con el proceso de contrarreforma. Esto nos permite verificar si este proceso se realizó en diferentes modos debido a las razones legales invocadas. Suponemos que la contrarreforma comienza en 1974 y que se enfocó en aquellos predios expropiados en los años anteriores a esa fecha. ${ }^{29}$

La tabla 10 presenta los resultados de este ejercicio, que relaciona la razón legal presentada para las expropiaciones con los respectivos resultados. El objetivo es estudiar si los predios expropiados en determinadas situaciones tuvieron destinos diferentes. Se aprecia que, para la mayor parte de las razones legales, la mayoría de las expropiaciones fueron distribuidas y luego una menor proporción fue revocada. La única razón legal que no sigue este patrón es la razón 4 (predios expropiados por ser explotados de forma ineficiente), donde el número de

${ }^{29}$ Es interesante destacar que la base de datos que hemos construido permite realizar otros análisis que no hemos reportado para ahorrar espacio. Por ejemplo, es posible estudiar el momento de la regularización del predio y su correlación con el momento y las razones invocadas para expropiar y con el mecanismo utilizado durante la contrarreforma. 
Tabla 10. RELACIÓN ENTRE RAZÓN LEGAL DE EXPROPIACIÓN Y RESULTADO FINAL DEL PROCESO DE REFORMA AGRARIA

PANEL A: PREDIOS

\begin{tabular}{|c|c|c|c|c|c|c|c|c|c|}
\hline \multicolumn{10}{|c|}{ Razones legales } \\
\hline Canal & 1 & 2 & 3 & 4 & 5 & 6 & 8 & 10 & 15 \\
\hline $\begin{array}{l}\text { Distri- } \\
\text { buido }\end{array}$ & 67 & 8 & 1.671 & 222 & 25 & 236 & 50 & 977 & 79 \\
\hline $\begin{array}{l}\text { Revoca- } \\
\text { do }\end{array}$ & 15 & 1 & 486 & 662 & 5 & 91 & 36 & 309 & 2 \\
\hline $\begin{array}{l}\text { Transfe- } \\
\text { rido }\end{array}$ & 2 & 0 & 42 & 18 & 0 & 21 & 1 & 24 & 1 \\
\hline $\begin{array}{l}\text { Subas- } \\
\text { tado }\end{array}$ & 2 & 0 & 45 & 25 & 0 & 11 & 2 & 24 & 0 \\
\hline $\begin{array}{l}\text { Restau- } \\
\text { rado }\end{array}$ & 0 & 0 & 9 & 13 & 0 & 1 & 0 & 4 & 0 \\
\hline Otro & 0 & 0 & 7 & 2 & 0 & 0 & 0 & 0 & 0 \\
\hline Total & 86 & 9 & 2.260 & 942 & 30 & 360 & 89 & 1.338 & 82 \\
\hline
\end{tabular}

PANEL B: SUPERFICIE (HF)

\begin{tabular}{lccccccccc}
\hline \multicolumn{7}{c}{ Razones legales } \\
\hline Canal & 1 & 2 & 3 & 4 & 5 & 6 & 8 & 10 & 15 \\
\hline $\begin{array}{l}\text { Distri- } \\
\text { buido }\end{array}$ & 10.797 & 8.981 & 1.559 .248 & 229.892 & 11.289 & 1.645 .240 & 22.045 & 1.086 .929 & 129.335 \\
$\begin{array}{l}\text { Revoca- } \\
\text { do }\end{array}$ & 1.606 & 140 & 1.198 .514 & 383.737 & 5.594 & 346.716 & 101.222 & 266.565 & 340 \\
$\begin{array}{l}\text { Transfe- } \\
\text { rido }\end{array}$ & 2.034 & 0 & 332.338 & 23.630 & 0 & 302.821 & 34.837 & 53.828 & 159.000 \\
$\begin{array}{l}\text { Subas- } \\
\text { tado }\end{array}$ & 975 & 0 & 130.649 & 20.797 & 0 & 140.361 & 2.598 & 148.332 & 0 \\
$\begin{array}{l}\text { Restau- } \\
\text { rado }\end{array}$ & 0 & 0 & 4.946 & 4.690 & 0 & 674 & 0 & 13.556 & 0 \\
Otro & 0 & 0 & 12.704 & 1.144 & 0 & 0 & 0 & 0 & 0 \\
\hline \begin{tabular}{l} 
Total \\
\hline
\end{tabular} & 15.412 & 9.120 & 3.238 .399 & 663.890 & 16.883 & 2.435 .811 & 160.701 & 1.569 .210 & 288.764 \\
\hline
\end{tabular}

predios revocados es mucho mayor que el número de predios distribuidos, lo que también es cierto cuando se observan las superficies involucradas. Una explicación sugerida por Bellisario (2007a; 2007b) es que el carácter discrecional de esta razón legal implicaba que se utilizó de manera arbitraria para justificar expropiaciones, lo que llevó a que des- 
pués de 1973 los antiguos propietarios impugnaran la medida. La investigación futura podría estudiar este fenómeno con detalles adicionales utilizando la nueva base de datos presentada en este artículo.

\section{COMENTARIOS FINALES}

En este trabajo hemos presentado una serie de hechos estilizados a partir de una nueva base de datos que permite examinar distintos aspectos de la reforma agraria chilena, que tuvo lugar entre 1962 y 1980. El conjunto de datos incorpora información a nivel de predio agrícola y de comuna para el período inicial de la reforma, el período de consolidación y el periodo de reversión (o contrarreforma). Además de los hechos estilizados, se correlaciona la intensidad de la reforma agraria a nivel comunal con los posibles factores asociados con la producción agrícola y la estructura inicial de propiedad de la tierra.

Estos hechos estilizados y la nueva base de datos deberían ser útiles para motivar la realización de una variedad de trabajos empíricos. Nuestros cálculos básicos proporcionan indicios razonables sobre la evolución y el alcance de la reforma a nivel de comuna, sobre las razones legales empleadas para justificar las expropiaciones y sobre el proceso de contrarreforma implementado por el régimen militar. La base de datos puede ser útil para el estudio de los vínculos de las comunas con diferentes proxies para el proceso de reforma e importantes variables económicas, políticas y sociales, tales como los niveles de producción, la desigualdad, el funcionamiento de las instituciones políticas locales, y los efectos de la reforma sobre resultados políticos (y viceversa). También puede ser posible utilizar las variables presentadas en la base de datos para estudiar la interacción de la reforma agraria con sus efectos sobre otras políticas públicas implementadas durante o después del proceso de reforma. ${ }^{30}$

Esperamos que este nuevo conjunto de información contribuya así a mejorar la calidad de la investigación y optimice las bases de la discusión sobre uno de los tópicos relevantes del siglo XX chileno.

\footnotetext{
${ }^{30}$ Un ejemplo de la utilización de esta base de datos es González (2013), quien estudia el impacto causal de la reforma inicial sobre resultados políticos.
} 


\section{REFERENCIAS}

Almonacid, F. 2009. La agricultura chilena discriminada (1910-1960). Una mirada de las políticas estatales y el desarrollo sectorial desde el sur. Madrid: CSIC.

Arnello, M. 2016. "A 50 años de la reforma agraria: mitos y realidad de un desastre". El Mercurio, 3 de marzo.

Assadourian, C. S. 2006. "Agriculture and Land Tenure". En The Cambridge Economic History of Latin America, Vol. I, editado por V. Bulmer-Thomas, J. Coatsworth \& R. Cortés-Conde, 275-314. Cambridge: Cambridge University Press.

Avendaño, O. 2014. "Los partidos frente a la cuestión agraria en Chile, 1967-1973”. Revista de Ciencia Política 52 (1): 93-122.

Baland, J. \& J. Robinson. 2008. "Land and Power: Theory and Evidence from Chile". American Economic Review 98 (5): 1.737-1.765.

Ballesteros, M. 1965. "Desarrollo agrícola chileno, 1910-1955". Cuadernos de Economía 2 (5): 1-40.

Banerjee, A. V. 1999. "Prospects and Strategies for Land Reforms". En Annual World Bank Conference on Development Economics 1999, editado por B. Pleskovic \& J. Stiglitz, 253-284. Washington, DC: World Bank.

Barahona, P., A. Valdés \& J. Quiroz. 1988. "Reformas económicas en la agricultura y respuesta de la producción agregada: Chile 1960-1987". Latin American Journal of Economics 25 (76): 391-404.

Barbier, P. 1984. "Inverse Relationship between Farm Size and Productivity. A Product of Science or Imagination?". Economic and Political Weekly 19 (5253): A189-A191, A193-A198.

Barraclough, S. 1970. “Agricultural Policy and Land Reform”. Journal of Political Economy 78 (4): 906-947.

Barraclough, S. 1999. "Land Reform in Developing Countries: The Role of the State and Other Actors". United Nations Research Institute for Social Development Discussion Paper 101.

Bauer, A. 1975. Chilean Rural Society: From the Spanish Conquest to 1930. Cambridge: Cambridge University Press.

Becket, J. 1963. "Land Reform in Chile”. Journal of Inter-American Studies 5 (2): $177-211$.

Bellisario, A. 2007a. "The Chilean Agrarian Transformation: Agrarian Reform and Capitalist 'Partial' Counter-Agrarian Reform, 1964-1980. Part 1: Reformism, Socialism and Free-Market Neoliberalism". Journal of Agrarian Change 7 (1): 1-34.

. 2007b. "The Chilean Agrarian Transformation: Agrarian Reform and Capitalist 'Partial' Counter-Agrarian Reform, 1964-1980. Part 2: CORA, Post-1980 Outcomes and the Emerging Agrarian Class Structure". Journal of Agrarian Change 7 (2): 145-182. 
2013. "La reforma agraria chilena. Reformismo, socialismo y neoliberalismo, 1964-1980". Historia Agraria 59: 159-190.

Bengoa, J. 2015. Historia rural de Chile central. Tomo II. Crisis y ruptura del poder hacendal. Santiago: LOM.

Brock. P. 2009. "Securitización de hipotecas y desarrollo económico: un ensayo sobre la Caja Hipotecaria de Chile". Economía Chilena 12 (1): 69-93.

Collier, S. \& W. Sater. 2004. A History of Chile, 1808-2002. Segunda edición. Cambridge: Cambridge University Press.

Cox, M. 2016. "La reforma agraria II”. El Mercurio, 5 de marzo.

Crespo, P. 2016. "La reforma agraria y sus lecciones". El Mercurio, 26 de abril.

Cuesta, J., F. Gallego \& F. González. 2015. "Local Impacts of Trade Liberalization: Evidence from the Chilean Agricultural Sector". En Economic Policies in Emerging-Market Economies, editado por R. Caballero \& K. Schmidt-Hebbel, 351-378. Santiago: Banco Central de Chile.

Díaz, J., R. Lüders \& G. Wagner. 2016. Chile 1810-2010. La república en cifras. Historical Statistics. Santiago: Ediciones Universidad Católica de Chile.

Edwards, S. 2009. "Forty Years of Latin America's Economic Development: From the Alliance for Progress to the Washington Consensus". NBER Working Paper 15190.

Fermandois, J. 2016. "Diez tesis sobre la reforma agraria". El Mercurio, 15 de marzo.

Fontaine, A. 2001. La tierra y el poder. Reforma agraria en Chile (1964-1973). Santiago: Zig-Zag.

Garrido, J. (editor). 1988. Historia de la reforma agraria en Chile. Santiago: Editorial Universitaria.

Gaurav, S. \& S. Mishra. 2015. "Farm Size and Returns to Cultivation in India: Revisiting an Old Debate". Oxford Development Studies 43 (2): 165-193.

Goic, P. \& E. Sanhueza. 1992. Eduardo Frei. Un camino en la dignificación del campesino. Santiago: Fundación Eduardo Frei.

Gómez, S. \& J. Echeñique. 1988. La agricultura chilena. Las dos caras de la modernización. Santiago: Flacso-Agraria.

González, F. 2013. "Can Land Reform Avoid a Left Turn? Evidence from Chile after the Cuban Revolution". The B.E. Journal of Economic Analysis \& Policy 13 (1): 31-72.

Hachette, D. 2011. Latinoamérica en el siglo XX. Crecimiento, comercio, pensamiento económico. Santiago: Ediciones Universidad Católica de Chile.

Huerta, M. 1989. Otro agro para Chile. La historia de la reforma agraria en el proceso social y politico. Santiago: Cisec-Cesoc.

Hurtado, H., E. Muchnik \& A. Valdés. 1990. Trade, Exchange Rate, and Agricultural Pricing Policies in Chile, Vol. I y II. Washington, DC: World Bank Comparative Studies. 
INE. 2007. División político-administrativa y censal. Disponible en: http://www. ine.cl/canales/chile_estadistico/territorio/division_politico_administrativa/pdf/ DPA_COMPLETA.pdf.

Kay, C. 1978. "Agrarian Reform and the Class Struggle in Chile". Latin American Perspectives 5 (3): 117-142.

Kay, C. \& P. Silva (editores). 1992. Development and Social Change in the Chilean Countryside: From the Pre-Land Reform Period to the Democratic Transition. Amsterdam: Cedla.

Krueger, A., M. Schiff \& A. Valdés. 1988. “Agricultural Incentives in Developing Countries: Measuring the Effect of Sectoral and Economy-wide Policies". World Bank Economic Review 2 (3): 255-272.

- 1991. The Political Economy of Agricultural Pricing Policy, Vol. 1: Latin America; Vol. 2: Asia; \& Vol. 3: Africa and the Mediterranean. Baltimore: Johns Hopkins University Press for the World Bank.

Lapp, N. 2004. Landing Votes: Representation and Land Reform in Latin America. New York: Palgrave Macmillan.

Lederman, D. 2005. The Political Economy of Protection: Theory and the Chilean Experience. Stanford, CA: Stanford University Press.

Loveman, B. 1976. Struggle in the Countryside. Politics and Rural Labour in Chile, 1919-1973. Bloomington: Indiana University Press.

- 2001. Chile. The Legacy of Hispanic Capitalism. Tercera edición. New York: Oxford University Press.

McBride, G. 1936. Chile: Land and Society. New York: American Geographical Society.

Meller, P. 1996. Un siglo de economía política chilena (1890-1990). Santiago: Andrés Bello.

Millar, R. \& J. Fernández. 2008. "Políticas agrarias en Chile: 1932-1958”. Boletín de la Academia Chilena de la Historia 74 (117): 407-464.

Moreno, R. 2013. Sin reforma agraria no habría sido posible. Memorias de la reforma agraria chilena 1958-1970. Santiago: Copygraph.

—. 2016. "A 50 años de la reforma agraria chilena". El Mercurio, 17 de febrero.

Portilla, B. 2000. "La política agrícola en Chile: lecciones de tres décadas". CEPAL, Serie Desarrollo Productivo 68.

PUC. 1976. El sector agrícola chileno: 1964-1974. Santiago: Pontificia Universidad Católica de Chile, Departamento de Economía Agraria.

Robles-Ortiz, C. 2009. "Agrarian Capitalism and Rural Labour: The Hacienda System in Central Chile, 1870-1920". Journal of Latin American Studies 41 (3): 493-526.

Santana, R. 2006. Agricultura chilena en el siglo XX: Contextos, actores y espacios agrícolas. Santiago: Dibam.

Sen, A. K. 1962. "An Aspect of Indian agriculture". Economic Weekly 14 (4-6): 243-246. 
Valdés, A. 1994. "Agricultural Reforms in Chile and New Zealand: a Review". Journal of Agricultural Economics 45 (2): 189-201.

2016a. "La reforma agraria". El Mercurio, 18 de abril.

. 2016b. "50 años de la reforma agraria: Algunas lecciones para el Chile de hoy". Revista Libertad y Desarrollo 275.

Valdés, A. \& E. Jara. 2008. "Chile”. En Distortions to Agricultural Incentives in Latin America, editado por K. Anderson \& A. Valdés, 119-158. Washington DC: The World Bank.

Valdés, A. \& W. Foster. 2014. "The Agrarian Reform Experiment in Chile History, Impact, and Implications". International Food Policy Research Institute Discussion Paper 01368.

- 2015. La reforma agraria en Chile: historia, efectos y lecciones. Santiago: Ediciones Universidad Católica de Chile e IFPRI.

Valdés, A., H. Hurtado \& E. Muchnik. 1990. "Chile”. En Economía política de las intervenciones de precios agrícolas en América Latina, editado por A. Krueger, M. Schiff \& A. Valdés, 145-204. Santiago: Banco Mundial-CINDE.

Wachter, S. 1979. "Structuralism vs. Monetarism: Inflation in Chile". En ShortTerm Macroeconomic Policy in Latin America, editado por J. Behrman \& J. Hanson, 227-256. Cambridge: NBER.

Williamson, C. 2016. "A 50 años de la reforma agraria chilena”. El Mercurio, 9 de febrero.

\section{ANEXO. DEFINICIÓN DE LAS UNIDADES GEOGRÁFICAS}

Las regiones utilizadas son las correspondientes a las definidas en 2007 y no tienen ningún problema de coincidencia con los datos más antiguos. Asignamos las unidades geográficas construidas a las regiones correspondientes y nunca se comparan directamente regiones de diferentes años

La tabla A1 muestra las regiones de 2007 con sus nombres correspondientes.

Como ya se explicó en la nota 18 , en cuanto a la definición de comuna que se utilizó a lo largo del trabajo se tuvo que lidiar con el problema de que las comunas varían entre 1955 y 2007 y, de hecho, el uso de las definiciones de 2007 conduciría a desajustes y a interpretaciones erróneas. A partir de este punto, lo que hicimos fue construir unidades geográficas que (i) tuviesen características similares y (ii) no cambiasen durante el período de estudio. En algunos casos, ambas condiciones fueron posible, pero en otros fue necesario agrupar hasta 7 comunas 
para elaborar una unidad que cumpliera tales condiciones. Este procedimiento implica que en lugar de las 346 comunas actuales sólo se utilizaron 271 unidades geográficas, pero haciendo posible comparaciones intertemporales.

Tabla A1. REGIONES DE CHILE, 2007

\begin{tabular}{ll}
\hline Número & Nombre \\
\hline I & Tarapacá \\
III & Antofagasta \\
IV & Atacama \\
V & Coquimbo \\
VI & Valparaiso \\
VII & O'Higgins \\
VIII & Maule \\
IX & Biobio \\
X & La Araucanía \\
XI & Los Lagos \\
XII & Aysén \\
XIII & de Magallanes y Antártica \\
\hline
\end{tabular}

